\title{
7. Über die Zerlegung der Spektrallinien von Barium, Yttrium, Zirkon und Osmium im magnetischen Felde; von Burton Evans Moore.
}

(Auszug aus der Göttinger Inaugural-Dissertation.)

\section{Finleitung.}

Die Untersuchungen von Runge ${ }^{1}$ ) und Paschen haben das Prestonsche Gesetz in mehrfacher Hinsicht bestätigt. In diesem Gesetze wurde eine enge Verwandtschaft zwischen den magnetischen Zerlegungen jener Spektrallinien, von denen man wuBte, daß sie Serien bilden, und ein verschiedener Charakter der Zerlegungen für verschiedene Serien behauptet; aber zugleich auch ein Grad von Ähnlichkeit zwischen den verschiedenen Serien. Für Barium sind bisher keine Tripletserien gefunden, die den Tripletserien in chemisch verwandten Substanzen $\mathrm{Mg}, \mathrm{Ca}, \mathrm{Sr}$ entsprechen. Statt nun zu versuchen, Prestons Gesetz durch Serien zu bestätigen, kann man nach der umgekehrten Methode verfahren und das Gesetz benutzen, um Serien zu bilden von den Linien gleicher magnetischer Zerlegung (diese selbst ausgedrückt durch das betreffende $\Delta \lambda / \lambda^{2}$, wo $\lambda$ die Wellenlänge, $1 / \lambda$ also die Wellenzahl pro Zentimeter und $\Delta \lambda$ die Entfernung einer Komponente von der normalen Lage ist). Bei der Anwendung dieses Verfahrens auf Barium wurden allerdings Vertreter der verschiedenen Arten Zerlegung gefunden, aber nicht genug Glieder, um Serien zu bilden. Die folgende Untersuchung versucht die letztere Methode zuerst für ein vollständigeres Studium des Bariums zu verwenden und dann auf andere Substanzen, für die bisher keine Serien gefunden sind, um schlieBlich $\mathrm{zu}$ sehen, ob eine Verwandtschaft $\mathbf{z w i s c h e n}$ den verschiedenen Zerlegungscharakteren oder -typen nachgewiesen werden kann.

1) C. Runge, Sitzungsber. der Berliner Akad. d. Wissensch. am 6. Febr. 1902 ; 19. p. 380. 1902; 19. p. 720. 1902; 20. p. 417. 1904; Astrophys. Journ. 15. p. 235, 333, 1902; 16. p. 123.1902. 


\section{Experimentelle Methode.}

Die experimentelle Methode ist im wesentlichen dieselbe, die Runge ${ }^{1}$ ) benutzt hat. Beim Versuche, das Zirkonspektrum zu photographieren, erhielt ich zuerst nur das Linienspektrum der Zinkelektroden und ein kontinuierliches Spektrum von Zirkon. Erst durch weitere Trennung der Pole und durch Verwendung von Gaskohlenplatten von $1 \mathrm{~mm}$ Dicke und $4 \mathrm{~mm}$ Breite als Elektroden erhielt ich einen Funken, der heiß genug war, um das Zirkon zu reduzieren. Dies neue Verfahren verdoppelte die Poldistanz und verringerte die Feldstärke wesentlich. Als Ersatz für diesen Verlust erzielte ich einen vielfach stärkeren Funken, der es mir ermöglichte, die Spaltbreite um die Hälfte zu reduzieren und viel stärkere und schärfere Linien bei weit kürzerer Expositionsdauer zu bekommen.

Die Länge der Expositionszeit variierte von $15 \mathrm{Min}$. bis zu 2 Stunden und bei Barium im rat bis zu 6 Stunden. Die längeren Expositionen wurden vorgenommen, um die schwachen Linien deutlich zu machen. Die Substanzen haften so sehr an den Kohlenelektroden, daß es möglich war, sehr kleine Mengen des Salzes zu verwenden. Dieser Umstand wird es möglich machen, einige der vielversprechenden selteneren Metalle zu studieren.

Die Photographien wurden alle bei gleicher Entfernung der Polschuhe gemacht; dabei konnte man durch Regulierung des Stromes im Elektromagneten nach Belieben jede Feldstärke unter dem Maximum erzielen. Die hauptsächlich gebrauchte Feldstärke war 24400 C.G.S.-Einh. Dies wurde aus wiederholten Messungen der Calciumlinie von der Wellenlänge $\lambda=3968,6 \AA$. .E. ermittelt, die als eine Unreinheit in der Kohlenstoffelektrode vorkommt und aus einigen speziellen Photographien der Zinklinie 4680; dabei wurde rorausgesetzt, daB Runges und Paschens Messungen dieser Linien sich auf eine Feldstärke von 31000 Einh. beziehen. Alle Resultate sind für die Feldstärke 24400 angegeben.

Andere Feldstärken wurden verwandt, um die wahren Komponenten sehr nahe beieinander befindlicher Linien zu bestimmen oder um Ablesungen zu bekommen für sonst sich

1) C. Runge, l. c. 
überdeckende Komponenten. Häufig wurden die Verschiebungen solcher Linien auch aus der Entfernung der einen wahrnehmbaren freien Komponente von der ungestörten Lage bestimmt.

Es wurden die Photographien von den Schwingungen, die parallel $(p)$ und von denen, die senkrecht $(s)$ zu den Kraftlinien stattfinden, je auf einer besonderen Reihe von Platten genommen. Dies wurde in der üblichen Weise vermittelst eines Calcitprismas bewirkt. Photographien ohne Calcit, d.h. sowohl mit den $p$ - als auch mit den $s$-Komponenten auf denselben Platten wurden nur verwendet, um die relative Intensität der $p$ - und $s$-Komponenten zu bestimmen. So weit die letzte Klasse von Platten in Frage kommt, sind die $p$ - und und $s$-Komponenten oft so nahe beieinander, daB dies ihre Zerlegung und die Kenntnis ihrer bezüglichen Intensitäten verhindert. Bei Tripletten von großer Zerlegung bot sie indessen keine Schwierigkeit.

Durch einen doppelten $\nabla$ ergleich solcher Linien mit entsprechenden Komponenten auf den $p$ - und den s-Platten lieB sich auch das Verhältnis der Intensitäten auf den $p$ - und den $s$-Platten feststellen. Die Linien konnten dann in der Tat auf den $s$ - und den $p$-Platten verglichen werden, obwohl die Expositions- und Entwickelungsbedingungen ganz verschiedene gewesen sein mögen. ${ }^{1}$ )

Immerhin ist ein recht groBer Fehler in der Bestimmung der Intensitäten möglich, einerseits, da ein großer Abstand in der Weite und Tiefe der Schwärzung besteht, andererseits, weil keine experimentelle Methode benutzt worden ist, um die Intensitäten $\mathrm{zu}$ vergleichen, wie dieses bei der Photometrie oder der Spektrophotometrie der Fall ist.

Die Verschiedenheit in der aktinischen Empfindlichkeit der Platten im ganzen Spektrum ist sehr grob, so daB man, um das ganze Spektrum zu beherrschen, zu verschieden präparierten Platten greifen muB. Auch waren die für rot empfindlichen Platten, selbstpräpariert mit Diacyamin, bei weitem nicht gleichförmig. Daher konnte ein Vergleich der Intensitäten von verschiedenen Linien, was bei der Aufsuchung von

1) Derartige Vergleichungen haben allerdings nach den neuesten Untersuchungen von Zeeman (Arch. Néerl, 1907, 28. Nov.) wenig all. gemeine Bedeutung. 
Serien sehr wichtig ist, selbst innerhalb eines kurzen Spektralbereiches nur annähernd genau sein. Dies ist meist ausreichend, um zu zeigen, daB zwei oder mehr einander nahe Linien gleicher magnetischer Zerlegung, aber von großer Ungleichheit in der Intensität, nicht zur selben Serie gehören. Dies ist aber alles, was man von den Intensitäten erwarten darf, die in den folgenden Tabellen angegeben werden. Wie groß die Veränderungen in der Stärke unter den verschiedenen Experimentalbedingungen sein können, kann man ersehen, wenn man die hier verzeichneten Intensitäten mit denen vergleicht, die in Exners und Hascheks Tabellen gegeben sind, welch letztere eingehend benutzt wurden. Es ist aber genug Ähnlichkeit vorhanden, um Exners und Hascheks Tabellen für die Identifizierung der Linien nutzbar zu machen.

Die Intensitäten, die unten mitgeteilt sind, beziehen sich auf die Linien erster Ordnung, ob nun die Linien in erster oder in zweiter Ordnung gemessen wurden. Die niedrigste Intensität, 1, ist nur eben bemerkbar. Die betreffende Linie ist nicht meBbar, ausgenommen, wenn sie sich günstig in einer Gruppe von Komponenten befindet und dann auch nicht besonders genau. Eine Linie von der nächsten Intensität, 2, kann gut gemessen werden in Gruppen von verschiedenen Komponenten, aber nicht in befriedigender Weise in einem Paare von breiter Zerlegung. Die folgende Intensität, 3, ist die niedrigste Intensität, die unter den letzten Bedingungen befriedigende Resultate liefert. Zerflossene Linien von höheren Intensitäten können gleichfalls unbefriedigend werden. Ein Paar Komponenten, die nach außen oder innen abgeschattet sind, weist auf das Vorhandensein äuBerer oder innerer schwacher Komponenten hin, die zur Darstellung gebracht werden können, wenn Selbstindnktion, Kapazität, Spaltbreite und Exposition der Platten gehörig ausgeglichen sind. Breite einfache Linien deuten auf eine ähnliche Zerlegung hin. Daher mag eine Feststellung dieser Tatsachen von Nutzen sein, sogar wenn die Zerteilung oder Zerlegung nicht bewirkt worden ist.

Die Genauigkeit der Ablesungen hängt natẗrlich auch von der Schärfe der Linien ab. Wo Schwäche, Zerflossenheit oder das Vorhandensein sich überdeckender Komponenten eine Ab- 
lesung weniger genau gemacht hat, ist das Resultat in Klammern eingeschlossen. Die eingeklammerten Werte sind immerhin noch genau genug, um eine ziemlich richtige Idee von der GröBe der Zerlegung zu geben.

Bezüglich der Verschiedenheiten der MeBgenauigkeit bei den verschiedenen untersuchten Spektren sowie einzelner Schwierigkeiten bei der Identifizierung von Linien nach den Exner-Haschekschen Tabellen muB auf die Dissertation verwiesen werden.

\section{Frklärung der Tabellen.}

Die Abkürzungen in den Tabellen haben die folgende Bedeutung: $\lambda$ Wellenlänge in A.E., $\Delta \lambda / \lambda^{2}$ die Änderung der Wellenzahl pro Zentimeter (die negativen und positiven Werte derselben liegen natürlich auf der roten bzw. blauen Seite der Ruhelagen). $s$ senkrecht, $p$ parallel zu den Kraftlinien stattfindende Schwingung. $H$ Hauptserie, $i$ Intensität, $g$ gröBere Wellenlänge, $k$ kleinere Wellenlänge, $N$ Nebenserie, $S$ Satellit, h Hauptlinie, $R$. Beobachtung nach Runge und Paschen, $M$. Beobachtung nach Moore.

Die mit $A$ überschriebene Spalte gibt den ungefähren Wert der Abstände der Komponenten, ausgedrückt durch einen kieinen konstanten Abstand, genannt das „Intervall“, letzteres je multipliziert mit kleinen ganzen Zahlen, genannt „Faktoren“. Die Faktoren stellen das Verhältnis der Entfernungen der sukzessiven Komponenten von der Lage der ungestörten Linie aus dar. $\left.{ }^{1}\right)$ Die mit $B$ überschriebene Spalte enthält die Bemerkungen. Insofern gleichlautende Bemerkungen häufig vorkommen, sind sie durch die folgenden Abkürzungen bezeichnet worden: $D$ zerstreut, $D_{r}$ zerstreut nach rot, $D_{b}$ zerstreut nach blau, $D_{i}$ und $D_{a}$ zerstreut nach innen und außen, was im allgemeinen auf das Vorhandensein ron schwachen inneren bez. äußeren Komponenten hinweist; $w$ wenig, $b$ stark verbreitet; $b_{0}$ und $r_{0}$ blaue bzw. rote Komponente überdeckt von der Komponente einer benachbarten oder fremden Linie; $n$. $i$. nicht identifiziert, d. h. die Linie kann nicht mit denen in Exners

1) Auf den Zusammenhang dieser Darstellang mit der von C. Runge (Physik. Zeitschr. 8. 15. April 1907) gegebenen komme ich weiter unten (auf p. 339) zurück.

Annalen der Physik. IV.Folge. 25. 
und Hascheks Tabellen verglichen werden. Spezielle Bemerkungen sind durch Zahlen angegeben, die am Schlusse der bezüglichen Tabellen erklärt werden.

In den Quadruplettabellen sind die zur Hauptlinie symmetrischen $\mathbf{z u}$ den Kraftlinien senkrechten und parallelen Komponenten jede durch ein doppeltes Zeichen $( \pm)$ bezeichnet, um Wiederholungen zu vermeiden. Die $s$-Komponente ist stets zuerst aufgeführt. Das doppelte Zeichen bedeutet daher zwei Ablesungen. Die tripletten $s$-Komponenten sind in ähnlicher Weise bezeichnet und die $p$-Nullkomponente ist auBer Betracht gelassen. Bei den Tripletten werden häufig zwei Intensitäten angegeben. Der zuerst verzeichnete Wert bezieht sich dann auf die $s$ - und der zweite auf die $p$-Komponente. Wenn drei Intensitäten für Tripletten angegeben sind, so sind die äußeren $p$-Komponenten ungleich intensiv. Die erste Ablesung gibt dann die rote, die dritte die blaue Komponente. Wo nur eine Intensitalt unter Tripletten angegeben ist, bezieht sie sich auf die $s$-Komponente und die $p$-Komponente hat die doppelte Intensität.

IV. Barium.

Die Bariumlinien für Wellenlängen, die kürzer als 5854 waren, wurden auf Platten gemessen, die von Runge und Paschen aufgenommen waren. Es wurde viel Zeit auf Versuche verwandt, stärkere Photographien von Barium zu erlangen, um die schwächeren Linien zur Darstellung zu bringen. Diese Linien waren entweder uberhaupt nicht auf den neuen Platten oder zu verschwommen, um befriedigende Messungen zu liefern. Einige der schärfsten Linien der neuen Platten wurden gemessen und mit den Messungen nach Runge und Paschens Platten in Übereinstimmung befunden. Es wurde daher für zwecklos erachtet, andere Linien nochmals zu messen. Diese Beobachtungen und die von Runge und Paschen bei Barium habe ich auf die Feldstärke von 24400 C.G.S.-Einh. reduziert, die (wie vorher gesagt) bei allen folgenden Messungen benutzt wurde. Die Photographie des roten Spektrums ergab alle Linien außer zweien in Kaisers und Runges Bogenspektrum und verschiedene neue Linien, von denen die meisten schwach sind. Die Wellenlängen dieser Linien wurden bestimmt aus ihren Entfernungen von bereits bekannten Linien. Die fort- 
währende Verwendung dieser selben Graduierung bei Zirkonund Yttriumlinien, zugleich mit Exners und Hascheks Tabellen, läßt vermuten, dab die Wellenlängen zuverlässig sind bis innerhalb der $0,1 \AA$.-Einh. Die Intensitäten für das rote Spektrum sind angegeben und zeigen die verhältnismäßigen Intensitäten für jenen Teil des Spektrums.

Die Tabelle $\mathrm{Ba}_{1}$ gibt die Werte $\Delta \lambda / \lambda^{2}$ für Linien, die schon von Runge und Paschen beobachtet sind und vom Verfasser nochmals gemessen wurden zur Vergleichung der erreichbaren Genauigkeit; Barium stellte sich dabei als die für die Messungen ungünstigste Substanz heraus von den vier Substanzen, die auf einen solchen Vergleich hin studiert wurden.

In Tabelle $\mathrm{Ba}_{2}$ hat die Linie 6675,3 Abstände ihrer Komponenten im Verhältnis von $0,635(0,1,3)$, die Komponenten der Linie 5997,4 können durch $0,55(1,2,3)$ dargestellt werden, die der Linie 5971,9 durch $0,30(2,5)$ und die der Linie 4580 möglicherweise durch $0,10(4,8,15)$.

Tabelle $\mathrm{Ba}_{3}$ enthält eine Liste von Tripletten, die in Gruppen mit gleicher Zerlegung angeordnet sind. Die ausgelassene $p$-Komponente hat die Stellung der Linie ohne Feld, d. h. mit Nullzerlegung. Die Abstände der Gruppen I, III und IV stehen in dem einfachen Verhältnis ron 3-2-1.

Die Abstände in Gruppe II unterscheiden sich von denen in Gruppe III um 1/11. Die Zerlegung in Gruppe III bezeichnet Runge als normales Triplett (siehe später unter VIII, Vergleichung der Substanzen), den bezüglichen Abstand mit $a$, die Abstände in diesen Gruppen sind daher 3a/2, $a$ und $a / 2$. Die Zerlegungen der Gruppen I, III, IV sind bei der Linie 5997,4 gleichzeitig vorhanden, ausgenommen die Nullkomponente, diejenige von Gruppe I kommt auch bei 4166, die von Gruppe II bei 5854 und von Gruppe III bei 4554 in Tabelle $B a_{1}$ vor.

In Tabelle $\mathrm{Ba}_{4}$ befinden sich die übrigen Bariumlinien und unter "Bemerkungen" sind die Linien mehrerer Komponenten angegeben, in denen Abstände aus den Tripletten vorkommen.

Aus diesen Tabellen ist zu ersehen, daB die Tripletten aussehen wie Systeme von mehreren Komponenten, in denen einige Komponenten fehlen, oder, was noch bedeutungsvoller ist: die Größen der Zerlegungen reduzieren sich auf wenige Abstände und diese kommen in verschiedenen Zerlegungstypen 
wieder. Die Triplettenlinien in Gruppe II haben dieselbe Zerlegung wie die mittlere Linie der ersten Nebenserie.

Tabelle $\mathrm{Ba}_{1}$.

\begin{tabular}{|c|c|c|c|c|c|}
\hline \multicolumn{2}{|c|}{$\begin{array}{c}\text { H. G. } \\
\lambda=4934\end{array}$} & \multicolumn{2}{|c|}{$\begin{array}{l}2 \mathbf{N} . \mathbf{K} . \\
\lambda=4525\end{array}$} & \multicolumn{2}{|c|}{$\begin{array}{l}2 \text { N. G. } \\
\lambda=4900\end{array}$} \\
\hline R. & M. & R. & M. & R. & M. \\
\hline$-1,44$ & $-1,45$ & $-1,42$ & $-1,44$ & $-1,75$ & $-1,83$ \\
\hline,- 74 &,- 72 &,- 76 &,- 72 & $-1,09$ & $-1,11$ \\
\hline,+ 73 &,+ 71 &,+ 75 &, 74 & -.36 &,- 35 \\
\hline$+1,45$ & $+1,46$ & 1,45 & 1,44 & $\begin{array}{l}+, 35 \\
+1,07 \\
+1,75\end{array}$ & $\begin{array}{l}+, 37 \\
+1,11 \\
+1,80\end{array}$ \\
\hline
\end{tabular}

\begin{tabular}{|c|c|c|c|c|c|}
\hline \multicolumn{2}{|c|}{$\begin{array}{c}\text { H. K. } \\
\lambda=4554\end{array}$} & \multicolumn{2}{|c|}{$\begin{array}{c}1 \text { N. K. } \\
\lambda=5854\end{array}$} & \multicolumn{2}{|c|}{$\begin{array}{l}1 \text { N. S. } \\
\lambda=4166\end{array}$} \\
\hline R. & M. & R. & M. & R. & M. \\
\hline$-1,75$ & $-1,83$ & $-1,76$ & $-1,76$ & $-1,63$ & - \\
\hline$-1,08$ & $-1,11$ & $-1,19$ & $-1,19$ & $-1,16$ & - \\
\hline,- 36 &,- 35 &,- 92 & - &,- 88 &,- 87 \\
\hline,+ 35 &,+ 37 &,+ 58 &,- 58 &,- 62 & - \\
\hline$+1,06$ & $+1,10$ &,+ 58 &,+ 57 &,+ 55 & - \\
\hline \multirow[t]{3}{*}{$+1,79$} & $+1,82$ &,+ 91 & - &,+ 90 &,+ 87 \\
\hline & & $+1,19$ & $+1,16$ & $+1,14$ & - \\
\hline & & $+1,76$ & $+1,74$ & $+1,70$ & - \\
\hline
\end{tabular}

Tabelle $\mathrm{Ba}_{2}$.

\begin{tabular}{|c|c|c|c|c|c|c|c|}
\hline \multicolumn{2}{|c|}{$\left.\lambda=6675,3^{1}\right)$} & \multicolumn{2}{|c|}{$\lambda=5997,4$} & \multicolumn{2}{|c|}{$\left.\lambda=5971,9^{2}\right)$} & \multicolumn{2}{|c|}{$\lambda=4580$} \\
\hline$i$ & $\Delta \lambda / \lambda^{2}$ & $i$ & $\Delta \lambda / \lambda^{2}$ & $i$ & $\Delta \lambda / \lambda^{2}$ & $i$ & $\Delta \lambda / \lambda^{2}$ \\
\hline 5 & $-1,90 s$ & 3 & $-1,64 s$ & 8 & $1,49 \mathrm{~s}$ & & $-1,54 \mathrm{~s}$ \\
\hline 1 & $-[, 64] p$ & 6 & $-1,10 p$ & 10 &, $61 p$ & &,$- 81 p$ \\
\hline $1+$ & $0 p$ & 3 &,$- 56 s$ & 10 &, $60 p$ & &,$- 38 s$ \\
\hline 1 & $+[, 63] p$ & 3 &,$+ 54 s$ & 8 & $1,49 \mathrm{~s}$ & & $+39 \mathrm{~s}$ \\
\hline 5 & $+1,90 s$ & 6 & $+1,10 p$ & & & &,$+ 81 p$ \\
\hline & & 3 & $+1,66 \mathrm{~s}$ & & & & $+1,46 s$ \\
\hline
\end{tabular}

1) Die $s$-Komponente von 6451 stimmt hiermit überein und möglicherweise auch die Parallelkomponeute, da letztere als ein breites, schwaches Band erseheint eher denn als eine einfache Linie.

2) Die $s$-Komponenten sind breit und können sich vielleicht in je zwei Linien auflösen. Sie sind allerdings am stärksten in der Mitte und dies ist gewöhnlich nicht der Fall bei Linien, die sich mit besserer Definition auflösen. 
Tabelle $\mathrm{Ba}_{3}$.

\begin{tabular}{|c|c|c|c|c|c|c|c|c|c|c|c|}
\hline \multicolumn{3}{|c|}{ Gruppe I } & \multicolumn{3}{|c|}{ Gruppe II } & \multicolumn{3}{|c|}{ Gruppe III } & \multicolumn{3}{|c|}{ Gruppe IV } \\
\hline$\lambda$ & $i$ & $\Delta \lambda / \lambda^{2}$ & $\lambda$ & $i$ & $\Delta \lambda / \lambda^{2}$ & $\lambda$ & $i$ & $\Delta \lambda / \lambda^{2}$ & $\lambda$ & $i$ & $\Delta \lambda / \lambda^{2}$ \\
\hline $\left.6694,4^{1}\right)$ & 5 & $\mp 1,66$ & $\left.6527,6^{2}\right)$ & 12 & $\mp 1,24$ & 6483,1 & 8 & 1,14 & 6653,7 & 4 &, 55 \\
\hline 6341,9 & 8 & 1,59 & 6148,6 & 3 & 1,17 & 6182,6 & 2 & 1,08 & 6611,8 & 8 &, 56 \\
\hline 4682 & & 1,65 & 6141,9 & 30 & 1,20 & $\left(6165,4^{2}\right)$ & 2 & 1,06 & 6433,5 & 6 &, 57 \\
\hline 4574 & & 1,64 & 5778 & & 1,22 & 6063,3 & 12 & 1,10 & 6019,7 & 10 &, 555 \\
\hline 4506 & & 1,67 & 4416 & & 1,18 & 5988,1 & & 1,09 & 4132,6 & &, 56 \\
\hline 4432 & & 1,64 & 4131 & & $1,18 R$ & 5826 , & & 1,12 & & & \\
\hline 4414 & & 1,62 & - & & $1,18 M$ & 5536 & & 1,11 & & & \\
\hline 3889,4 & & 1,68 & $3993,2)$ & & 1,24 & 4727 & & 1,11 & & & \\
\hline & & & 3071,7 & & 1,17 & 4283 & & 1,07 & & & \\
\hline & & & & & & 3935 , & & 1,09 & & & \\
\hline & & & & & & 3501 , & & 1,11 & & & \\
\hline
\end{tabular}

Tabelle $\mathrm{Ba}_{4}$.

\begin{tabular}{|c|c|c|c|c|c|c|c|}
\hline$\lambda$ & $i$ & $\Delta \lambda / \lambda^{2}$ & Bemerkung & $\lambda$ & $i$ & $\Delta \lambda / \lambda^{2}$ & Bemerkung \\
\hline 6687,5 & & & Zu schwache Triplette & 6409,3 & 6 & -- & Breit, nicht sichtbar \\
\hline 6630,5 & & & " & & & & zerlegt \\
\hline $\left.6595,55^{3}\right)$ & 10 & $\mp, 635$ & Vgl. 5854 & 6403,1 & 4 & $\mp, 84$ & \\
\hline 6548,3 & 2 &, 63 & $\begin{array}{l}\text { (Nicht Cu 3274,1, die } \\
\text { eine Zerlegung von } \\
1,50 \text { hat.) }\end{array}$ & 6200,3 & & & $\begin{array}{l}\text { Breit, sieht wie mel- } \\
\text { rere Komponenten } \\
\text { aus } \\
\text { Zu schwach. Triplett }\end{array}$ \\
\hline 6498,9 & 8 & 1,48 & & 6192,1 & 3 &, 89 & Vgl. 5854 \\
\hline 6497,1 & 20 & 93 & & 6132,2 & 3 & ,88 & $"$ \\
\hline 6495,3 & 2 & ,68 & $\begin{array}{l}\text { (Nicht Cu } 3247,66 \text {, die } \\
\text { eine Zerlegung von } \\
1,10 \text { hat.) }\end{array}$ & $\begin{array}{l}6111,0 \\
5519\end{array}$ & 10 & $\begin{array}{l}1,28 \\
1,02 \\
1,2\end{array}$ & $8 a / 7 ?$ \\
\hline $\left.6484,7^{3}\right)$ & 6 &, 93 & $\begin{array}{l}\text { (Nicht Yt } 3242,43, \text { die } \\
\text { eine Zerlegung von }\end{array}$ & 4600 & & $\begin{array}{l}1,53 \\
1,28\end{array}$ & Vgl. 4580 \\
\hline $\left.6451,0^{4}\right)$ & 3 & 1,89 & 1,33 hat.) & $\begin{array}{l}4350, \\
3996,\end{array}$ & & $\begin{array}{l}1,83 \\
1,38\end{array}$ & Vgl. 4554 \\
\hline 6435,2 & 4 & 1,29 & $8 a / 7 ?$ & $\begin{array}{l}3910 \\
3892\end{array}$ & & $\begin{array}{l}, 84 \\
, 87\end{array}$ & \\
\hline
\end{tabular}

1) Die $s$-Komponenten sind jede ungefähr doppelt so stark wie die $p$-Komponente. In den anderen Linien sind die parallelen Komponenten die stärkeren Linien.

2) Gehört möglicherweise nicht zu dieser Gruppe.

3) Die s-Komponente ist ungefähr doppelt so intensiv wie die $p$-Komponente.

4) Vgl. die Anmerkung 1, Tabelle $\mathrm{Ba}_{2}$. 


\section{Yttrium.}

Die beiden folgenden Linien haben zwölf bez. zehn Komponenten.

\begin{tabular}{|c|c|c|c|c|c|}
\hline \multicolumn{3}{|c|}{$\lambda=3818,49$} & \multicolumn{3}{|c|}{$\lambda=4235,89$} \\
\hline$i$ & $\Delta \lambda / \lambda^{2}$ & $A$ & $i$ & $\Delta \lambda / \lambda^{2}$ & A \\
\hline 2 & $-1,74 \mathrm{~s}$ & $4 \times, 433$ & 2 & $-[1,26] \mathrm{s}$ & $7 \times, 18$ \\
\hline 4 & $-1,29 \mathrm{~s}$ & 3 & 2 & $-[, 89] \mathrm{s}$ & \\
\hline 10 &,$- 88 p$ & 2 & 6 &,$- 71 p$ & 4 \\
\hline 3 &,$- 85 s$ & 2 & 2 & $-[, 54] s$ & 3 \\
\hline 2 &,$- 44 p$ & 1 & 2 &,$- 36 p$ & 2 \\
\hline 2 &,$- 42 s$ & 1 & 2 &,$+ 36 p$ & \\
\hline 2 &,$+ 42 s$ & & - & - & \\
\hline 2 &,$+ 43 p$ & & 6 &,$+ 71 p$ & \\
\hline 3 &,$+ 84 s$ & & - & - & \\
\hline 10 &,$+ 88 p$ & & - & - & $b_{0}$ \\
\hline 3 & $+1,33 s$ & & & & \\
\hline 2 & $+1,74 s$ & & & & \\
\hline
\end{tabular}

Die folgenden fünf Linien haben jede neun Komponenten.

\begin{tabular}{|c|c|c|c|c|c|c|c|c|}
\hline \multicolumn{3}{|c|}{$\lambda=4398,21$} & \multicolumn{3}{|c|}{$\lambda=4199,46$} & \multicolumn{3}{|c|}{$\lambda=3950,51$} \\
\hline$i$ & $\Delta \lambda / \lambda^{2}$ & $A$ & $i$ & $\Delta \lambda / \lambda^{2}$ & $A$ & $i$ & $\Delta \lambda / \lambda^{2}$ & $A$ \\
\hline $1+$ & $-[1,72] s$ & $9 \times, 19$ & 6 & $-2,73 s$ & $5 \times, 54$ & 8 & $-1,51 \mathrm{~s}$ & $3 \times, 50$ \\
\hline 2 & $-1,33 s$ & & 3 & $-1,58 s$ & & 4 &,$- 98 \mathrm{~s}$ & \\
\hline 6 &,$- 95 s$ & 5 & 6 & $-1,02 p$ & 2 & 20 &,$- 51 p$ & 1 \\
\hline 4 &,$- 38 p$ & 2 & $1+$ &,$- 54 \mathrm{~s}$ & 1 & 2 &,$- 49 \mathrm{~s}$ & 1 \\
\hline 6 & $0 p$ & 0 & 10 & $0 p$ & 0 & 15 & 0 & 0 \\
\hline 4 &,$+ 38 p$ & & $1+$ &,$+ 58 s$ & & 2 &,$+ 49 s$ & \\
\hline 6 &,$+ 95 \mathrm{~s}$ & & 6 & $+1,02 p$ & & 20 &,$+ 49 p$ & \\
\hline 2 & $+1,34 s$ & & 3 & $+1,61 s$ & & 4 & $+1,02 \mathrm{~s}$ & \\
\hline $1(?)$ & - $s$ & & 6 & $+-2,73 s$ & & 8 & $+1,51 \mathrm{~s}$ & \\
\hline
\end{tabular}

\begin{tabular}{r|c|c||r|r|r}
\hline \multicolumn{3}{c||}{$\lambda=3628,89$} & \multicolumn{3}{c}{$\lambda=3584,71$} \\
\hline \multicolumn{1}{c|}{$\lambda$} & $\Delta \lambda / \lambda^{2}$ & $A$ & $i$ & $\Delta \lambda / \lambda^{2}$ & $A$ \\
\hline \hline 10 & $-1,92 s$ & $3 \times, 63$ & 12 & $-2,07 s$ & $8 \times, 265$ \\
5 & $-1,27 s$ & 2 & 6 & $-1,32 s$ & 5 \\
8 &,$- 66 p$ & 1 & 10 &,$- 76 p$ & 3 \\
2 &,$- 64 s$ & 1 & 3 &,$- 55 s$ & 2 \\
10 & $0 p$ & 0 & 15 & 0 & 0 \\
2 &,$+ 62 s$ & & 3 &,$+ 53 s$ & \\
8 &,$+ 66 p$ & & 10 &,$+ 74 p$ & \\
5 & $+1,28 s$ & & 6 & $+1,31 s$ & \\
10 & $+1,92 s$ & & 12 & $+2,07 s$ &
\end{tabular}


In der Linie 4398,2 kommt eine gemeinsame Differenz zwischen Komponenten des Wertes ,38 sechsmal vor. Das Intervall bei 4199,46 erscheint als das doppelte des Intervalls bei 3584,71 , oder die beiden Linien können beide das kleinere Intervall in verschiedenen Proportionen haben. Bei jeder der Linien 3950,5 und 3628,89 kommt der Fall vor, daB die $s$ und die $p$.Komponenten die gleiche Lage haben.

Die beiden folgenden Linien haben je acht Komponenten.

\begin{tabular}{c|c|l||l|l|l}
\hline \hline \multicolumn{3}{c|}{$\lambda=4236,10$} & \multicolumn{3}{c}{$\lambda=4083,89$} \\
\hline$i$ & $4 \lambda / \lambda^{2}$ & $A$ & $i$ & $\Delta \lambda / \lambda^{2}$ & $A$ \\
\hline \hline \multirow{2}{*}{6} & $-1,96 s$ & $9 \times, 22$ & 2 & {$[-1,79] s$} & $9 \times, 20$ \\
2 & $-1,53 s$ & 7 & 3 & $-1,19 s$ & 6 \\
3 &,$- 65 p$ & 3 & 6 &,$- 83 p$ & 4 \\
3 &,$- 22 p$ & 1 & $1+$ &,$- 64 s$ & 3 \\
3 &,$+-p$ & & 2 &,$+ 62 s$ & \\
3 &,$+-p$ & & 6 &,$+ 83 p$ & \\
2 & $-1,53 s$ & & 3 & $+1,19 s$ & \\
6 & $-1,96 s$ & & $1+$ & {$[+1,75] s$} &
\end{tabular}

Die drei folgenden Linien sind Sextette.

\begin{tabular}{r|c|l|r|r|r||r|r|r}
\hline \hline \multicolumn{3}{c|}{$\lambda=4358,91$} & \multicolumn{3}{c||}{$\lambda=3747,70$} & \multicolumn{3}{c}{$\lambda=3195,80$} \\
\hline$i$ & $\Delta \lambda / \lambda^{2}$ & $A$ & \multicolumn{1}{c|}{$i$} & $\Delta \lambda / \lambda^{2}$ & $A$ & $i$ & $\Delta \lambda / \lambda^{2}$ & $A$ \\
\hline \hline 12 & $-1,65 s$ & $3 \times, 553$ & 6 & $-1,04 s$ & & 8 & $-1,69 s$ & $3 \times, 553$ \\
15 & $-1,10 p$ & 2 & 6 &,$- 52 s$ & & 12 & $-1,11 p$ & 2 \\
12 &,$- 54 s$ & 1 & 12 &,$- 46 p$ & & 8 &,$- 55 s$ & 1 \\
12 &,$+ 54 s$ & & 12 &,$+ 46 p$ & & 8 &,$+ 55 s$ & \\
15 & $+1,10 p$ & & 6 &,$+ 52 s$ & & 12 & $+1,11 p$ & \\
12 & $+1,65 s$ & & 6 & $+1,04 s$ & & 8 & $+1,69 p$ &
\end{tabular}

Hier sind zwei Linien, 4358,91 und 3195,80 genaue Duplikate voneinander. Eine solche Übereinstimmung gilt im allgemeinen als die Andeutung des Vorhandenseins einer Serie. Leider sind in den Grenzen dieser Beobachtungen andere Glieder nicht vorhanden. Die $s$-Komponenten sind von gleicher Intensität und symmetrischer Lage. Sie sind von der Null- 
position um das ein- und dreifache des Wertes $\mp, 55$ entfernt. Die $p$-Komponenten entsprechen dem zweifachen desselben Wertes.

Bei 3747,70 haben die Abstände der $s$-Komponenten den einfachen und den doppelten Wert 干,52. Ein direkter Vergleich der $s$ - und der $p$-Platten zeigt, daB die gemessene Differenz der $p$-Komponenten und der inneren $s$-Komponenten tatsächlich rorhanden ist und nicht auf einem Beobachtungsfehler beruht. Das p. 313 erörterte Gesetz für die Abstände würde auch hier gelten, wenn man so weit gehen wollte, die kleine Differenz $z$ wischen diesen parallelen und inneren senkrechten Komponenten als Einheit zu nehmen. Die Abstände des Sextettes 4747,70 wären dann der 7,8- und 16 fache Wert von $\mp, 065$.

Die folgenden zwei Quintette waren zu genauen Messungen nicht geeignet.

\begin{tabular}{c|c|c||c|c|c}
\hline \multicolumn{3}{c||}{$\lambda=4477,1$} & \multicolumn{3}{c}{$\lambda=3941,76$} \\
\hline$i$ & $\Delta \lambda / \lambda^{2}$ & $B$ & $i$ & $\Delta \lambda / \lambda^{2}$ & $B$ \\
\hline \hline \multirow{2}{*}{2} & $-1,89 s$ & $5 \times, 38$ & 6 & $-1,47 s$ & $4 \times, 38$ \\
- & $-p$ & & $1+$ & $-[0,38] p$ & 1 \\
- & $-p$ & & 2 & $0 \quad p$ & 0 \\
- & $-p$ & & $1+$ & $+[0,38] p$ & \\
2 & $-1.89 s$ & & 6 & $+1,47 s$ &
\end{tabular}

Die $p$-Komponenten sind in dem Spektrum erster Ordnung nicht getrennt. So weit man in zweiter Ordnung sehen konnte, sind sie dieselben für beide Linien und können durch Null und den einfachen Wert $\mp, 38$ dargestellt werden. Die $s$-Komponenten von 4477,10 entsprechen dem Fünffachen dieses Wertes, bei 3951,76 dem Vierfachen. Die s-Komponenten von 3951,76 sind nach innen diffus und lassen ein Linienpaar von dem Werte $\Delta \lambda / \lambda^{2}=\mp 1,30$ vermuten, was dem Siebenfachen von ,19 entsprechen würde. Dieses bedeutet nur eine Verdoppelung der oben angegebenen multiplen Faktoren. 
Die folgenden vierzehn Linien sind Quadrupletts. 4477,59 ist anscheinend gleichfalls ein Quadruplett, aber zu schwach und zu diffus zum Messen.

\begin{tabular}{|c|c|c|c|c|c|c|c|c|c|}
\hline$\lambda$ & $i$ & $\Delta \lambda / \lambda^{2}$ & $A$ & $B$ & $\lambda$ & $i$ & $\Delta \lambda / \lambda^{2}$ & $A$ & $B$ \\
\hline 5510,1 & $\begin{array}{l}2+ \\
3\end{array}$ & $\begin{array}{l}\mp, 85 s \\
\mp, 48 p\end{array}$ & $7 \times \pm, 12$ & & $4 \mathbf{1 0 6}, 60$ & $\begin{array}{l}5 \\
3\end{array}$ & $\overline{40}$ & & $b_{0}$ \\
\hline 4682,5 & $\begin{array}{l}12 \\
10\end{array}$ & $\begin{array}{l}1,40 \mathrm{~s} \\
1,19 p\end{array}$ & $7 x+20$ & & 3982,75 & $\begin{array}{l}60 \\
60\end{array}$ & $\begin{array}{r}1,17 \\
, 53\end{array}$ & $11 \times, 105$ & $\left.D_{r}^{2}\right)$ \\
\hline 4475,90 & $\begin{array}{l}2 \\
3\end{array}$ & $\begin{array}{c}\lfloor 1,44] \\
, 52\end{array}$ & $\begin{array}{l}3 \times \pm, 50 \\
1\end{array}$ & & 3930,84 & $\left|\begin{array}{c}5 \\
6-44\end{array}\right|$ & $\begin{array}{r}1,21 \\
, 34\end{array}$ & $\begin{array}{l}4 \times, 30 \\
1 .\end{array}$ & \\
\hline 4375,11 & $\begin{array}{l}100 \\
100\end{array}$ & $\begin{array}{r}1,07 \\
, 20\end{array}$ & $\begin{array}{l}5 \times \pm, 20 \\
1\end{array}$ & & 3833,10 & $\begin{array}{l}30 \\
25\end{array}$ & $\begin{array}{r}1,34 \\
, 70\end{array}$ & $\begin{array}{l}2 \times, 67 \\
1\end{array}$ & \\
\hline 4177,68 & $\begin{array}{l}100 \\
100\end{array}$ & $\begin{array}{r}1,00 \\
, 44\end{array}$ & $\mid \begin{array}{l}5 x \pm, 20 \\
2\end{array}$ & $\begin{array}{l}D_{r} b \\
D_{r} b\end{array}$ & 3552,87 & $\overline{5}$ & $\overline{, 895}$ & & $r_{0}$ \\
\hline 4167,65 & $\begin{array}{l}20 \\
20\end{array}$ & $\begin{array}{r}1,20 \\
, 59\end{array}$ & $\begin{array}{l}6 \times \pm, 20 \\
3\end{array}$ & 1) & 3448,98 & $\begin{array}{l}6 \\
8\end{array}$ & $\begin{array}{r}1,42 \\
, 55\end{array}$ & $\begin{array}{l}3 \times, 50 \\
1\end{array}$ & 1) \\
\hline 4128,49 & $\begin{array}{l}30 \\
30\end{array}$ & $\begin{array}{l}\mp 1,26 s \\
\mp \quad, 29 p\end{array}$ & $4 \times \quad, 30$ & $\begin{array}{l}D_{r} w \\
D_{r} w\end{array}$ & 3200,44 & $\begin{array}{l}20 \\
20\end{array}$ & $\begin{array}{r}1,53 \\
, 69\end{array}$ & $\begin{array}{l}4 \times, 37 \\
2\end{array}$ & \\
\hline
\end{tabular}

Die Linien 4475,90 und 3448,98 sind in ihrem Verhalten einander sehr ähnlich, aber sie gehören nicht zur selben Serie, insofern als die blaue Linie die viel stärkere ist. Der Charakter der Zerlegung ist ferner ziemlich gleich bei 4128,49 und 3930,84 . Die Intensitäten stehen jedoch auBer allem Verhältnis zu dem auf Grund der Seriengesetze zu erwartenden. Sie sind so, wie man sie bei zwei parallelen Serien erwarten könnte.

Die folgenden 74 Linien sind Tripletten. Die Intensitäten der ungestörten $p$-Komponenten sind nicht angegeben. Das Verhältnis der Intensität der $s$ - zur $p$-Komponente ist gewöhnlich eins zu zwei, obwohl in vielen Fällen das Verhältnis zwei zu fünf näher kommt.

1) Die Breite der $s$-Komponenten läBt zwei oder mehr Komponenten vermuten.

2) Wenn die $p$ - und die $s$-Komponenten dieser starken Linie unter dem Mikroskope in Juxtaposition gebracht werden, stehen die Komponenten offenbar nicht in dem Verhältnis von zwei zu eins. Das Verhältnis zwölf zu fünf mal $a / 11$ (siehe später) scheint wahrscheinlicher, aber das hier gegebene Verhältnis elf zu fïnf paBt besser sowohl bei den alten wie den neuen Ablesungen. 


\begin{tabular}{r|c|r||r|r|r}
\hline \hline$\lambda$ & $i$ & $\Delta \lambda / \lambda^{2}$ & \multicolumn{1}{c|}{$\lambda$} & \multicolumn{1}{c|}{$i$} & $\Delta \lambda / \lambda^{2}$ \\
\hline 5663,1 & 12 & $\mp 1,10$ & 4840,1 & 3 & $\mp 1,50$ \\
30,3 & 2 & {$[, 74]$} & 4675,01 & 20 & 1,20 \\
5582,1 & 4 &, 97 & 43,88 & 25 & 1,02 \\
27,8 & $4-6$ & 1,11 & 4527,98 & 6 & 1,32 \\
5497,6 & 6 & 1,65 & 27,43 & 8 & 1,37 \\
66,7 & 8 & 1,03 & 06,12 & 15 &, 92 \\
03,0 & 6 &, 79 & 4465,50 & 35 & 1,06 \\
5205,9 & 10 & 1,18 & 46,85 & 3 & 1,42 \\
00,6 & 6 &, 74 & 43,83 & $1+$ & - \\
5087,6 & 10 & 1,34 & 22,80 & 25 &, 54 \\
4956,7 & $2+$ &, 48 & 4348,91 & 8 & 1,27 \\
4900,3 & 20 & 1,10 & 30,85 & 2 &, 84 \\
4883,9 & 20 & 1,24 & 09,81 & 50 & 1,33 \\
4855,1 & 15 &, 92 & & & \\
\hline \hline
\end{tabular}

\begin{tabular}{|c|c|c|c|c|c|c|c|}
\hline$\lambda$ & $i$ & $d \lambda / \lambda^{*}$ & $B$ & $\lambda$ & $i$ & $\Delta \lambda / \lambda^{2}$ & $B$ \\
\hline 4302,45 & 8 & $=1,13$ & & 3645,67 & 8 & $F 1,50$ & $n i$ \\
\hline 4251,39 & 6 & 1,09 & & 35,60 & 5 & 1,16 & \\
\hline 11,85 & 10 & 1,21 & & 33,28 & 50 & 1,02 & $D_{r}$ \\
\hline 04,84 & 20 & 1,61 & & 21,12 & 15 & 1,14 & \\
\hline 4174,27 & 30 & 1,25 & & 14,81 & $1+$ & {$[1,24]$} & \\
\hline 43,01 & 30 &, 91 & $D_{r} w$ & 11,10 & 60 & 1,30 & \\
\hline 25,10 & 20 & 1,35 & & 02,12 & 40 & ,62 & \\
\hline 02,60 & 50 & 1,23 & & 00,90 & 75 & 1,48 & \\
\hline 4077,54 & 50 & 1,21 & & 3593,11 & 8 &, 91 & \\
\hline 47,81 & 20 &, 86 & & 87,86 & 5 & 1,12 & \\
\hline 40,00 & 15 & 1,57 & $D_{i}$ & 85,90 & 3 & - & $r_{0}$ \\
\hline 3967,74 & 6 & 1,12 & & 49,21 & 50 & 1,67 & \\
\hline 44,90 & 7 & 1,34 & $n i$ & 31,85 & 5 & 1,21 & \\
\hline 06,57 & 5 & 1,48 & & 3496,25 & 12 & ,62 & \\
\hline 3878,80 & 15 & 2,34 & $n i$ & 68,05 & 3 &, 62 & \\
\hline 42,00 & 5 & 1,22 & $n i$ & 3372,93 & 6 & 1,25 & \\
\hline 3788,88 & 50 & 1,02 & $D_{r} w$ & 62,20 & 10 & 1,28 & \\
\hline 82,50 & 15 & 1,38 & & 28,11 & 30 & 1,08 & \\
\hline 76,73 & 15 & 1,49 & & 20,10 & $1+$ & {$[1,26]$} & $D$ \\
\hline 74,51 & 100 & 1,15 & $D_{r} w$ & 3242,49 & 30 & 1,33 & $D$ \\
\hline 10,41 & 60 & 1,25 & & 16,87 & 15 & 1,12 & $D_{a}$ \\
\hline 3696,90 & 4 & 85 & $D$ & 03,51 & 12 &, 53 & \\
\hline 68,67 & 6 & 1,38 & & 3173,40 & 5 & 1,23 & \\
\hline 64,76 & 30 & 1,74 & $D_{r} w$ & 30,20 & $3-2$ & 1,25 & \\
\hline
\end{tabular}

1) $Y 3878,47$ ist nicht vorhanden, falls es nicht diese Linie sein sollte, was kaum glaublicb erscheint. 
Jeder Abstand in der Triplettenklasse ist ein kleines Vielfaches eines der Intervalle, die bei Linien mit mehr Komponenten gefunden sind. Viele Linien könnten indessen verschiedene Vielfache von mehr als einem dieser Werte sein. Es ist deshalb von keiner Bedeutung, die Linien nach diesem Prinzip zu klassifizieren.

VI. Zirkon.

Die folgenden beiden Linien haben je elf Komponenten.

\begin{tabular}{c|c|c||c|c|c}
\hline \multicolumn{3}{c}{$\lambda=3573,30$} & \multicolumn{3}{c}{$\lambda=3272,39$} \\
\hline$i$ & $\Delta \lambda \lambda^{2}$ & $A$ & $i$ & $A \lambda / \lambda^{2}$ & $A$ \\
\hline \hline 5 & $-2,24 s$ & $6 \times, 37$ & 5 & $-2,26 s$ & $6 \times, 37$ \\
3 & $-1,50 s$ & 4 & 2 & $-1,54 s$ & 4 \\
3 & $-1,11 p$ & 3 & 3 & $-1,11 p$ & 3 \\
1 &,$- 74 s$ & 2 & 1 &,$-s$ & 2 \\
6 &,$- 34 p$ & 1 & 5 &,$- 37 p$ & 1 \\
1 &,$-s$ & 0 & 1 &,$-s$ & 0 \\
6 &,$+ 34 p$ & & 5 &,$+ 37 p$ & \\
1 &,$-s$ & & 1 &,$+-p$ & \\
3 & $+1,03 p$ & & 3 & $+1,14 p$ & \\
3 & $+1,50 s$ & & 2 & $+1,50 s$ & \\
$?$ &,$+-s$ & $b_{0}$ & 5 & $+2,26 s$. &
\end{tabular}

Diese beiden Linien sind sicher Duplikate. Die s-Komponenten sind gerade Vielfache von 0,37 und die $p$-Komponenten ungerade Vielfache desselben Wertes. Die allgemeine Differenz 0,74 kommt sechsmal in jeder dieser Linien vor.

Die folgenden beiden Linien haben je neun Komponenten.

\begin{tabular}{c|c|c||c|c|c}
\hline \multicolumn{3}{c||}{$\lambda=3780,78$} & \multicolumn{3}{c}{$\lambda=3921,99$} \\
\hline$i$ & $\Delta \lambda / \lambda^{2}$ & $A$ & $i$ & $\Delta \lambda / \lambda^{2}$ & $A$ \\
\hline \hline \multirow{2}{*}{6} & $-2,17 s$ & $5 \times, 43$ & 4 & $-2,14 s$ & $5 \times, 43$ \\
2 & $-1,69 s$ & 4 & 1 &,- & - \\
2 &,$- 86 p$ & 2 & 2 &,$- 92 p$ & 2 \\
3 &,$- 42 p$ & 1 & 3 &,$- 44 p$ & 1 \\
3 & $0 p$ & 0 & 3 & $0 p p$ & 0 \\
3 &,$+ 48 p$ & & 2 &,$+ 46 p$ & \\
$1+$ &,$+ 90 p$ & & $1+$ &,$+ 91 p$ & \\
2 & $+1,69 s$ & & 1 &,- & \\
6 & $+2,17 s$ & & 4 & $-2,14 s$ &
\end{tabular}


Diese Linien sind Duplikate und die Zerlegungen sind alle Vielfache des Wertes 0,43 .

Die sechs folgenden Linien haben je acht Komponenten.

\begin{tabular}{|c|c|c|c|c|c|}
\hline \multicolumn{3}{|c|}{$\lambda=4440,80$} & \multicolumn{3}{|c|}{$\lambda=4268,22$} \\
\hline$i$ & $\Delta \lambda / \lambda^{2}$ & $A$ & $i$ & $\Delta \lambda / \lambda^{2}$ & $A$ \\
\hline 2 & $-2,14 s$ & & 10 & $-2,16 s$ & $4 \times, 54$ \\
\hline 4 & $-1,51 \mathrm{~s}$ & & 8 & $-1,09 \mid p$ & 一 \\
\hline 2 & $-1,04 s$ & & 6 & $-1,07\} s$ & 2 \\
\hline 15 &,$- 89 p$ & & $3 s$ & 0 & \\
\hline 15 &,$+ 89 p$ & $9 \times, 10$ & $12 p$ & 0 & \\
\hline 2 &,$+ 98 s$ & 10 & 8 & $1,07\} p$ & \\
\hline 4 & $+1,57 \mathrm{~s}$ & 16 & 6 & $1,12\} \mathrm{s}$ & \\
\hline 2 & $+2,18 s$ & 22 & 10 & $2,16 s$ & \\
\hline
\end{tabular}

\begin{tabular}{c|c|l||l|l|l}
\hline \multicolumn{3}{c||}{$\lambda=4214,05$} & \multicolumn{3}{c}{$\lambda=4027,4$} \\
\hline$i$ & $\Delta \lambda / \lambda^{2}$ & $A$ & $i$ & $\Delta \lambda / \lambda^{2}$ & $A$ \\
\hline \multirow{2}{*}{6} & $-1,76 s$ & $3 \times, 60$ & 2 & $-1,94 s$ & $7 \times, 27$ \\
6 & $-1,21 p$ & 2 & 3 & $-1,36 s$ & 5 \\
2 & $-1,17 s$ & 2 & 8 & $-1,09 p$ & 4 \\
$10 p$ & & 0 & 2 &,$- 75 s$ & 3 \\
$1 s$ & 0 & & 2 &,$+ 87 s$ & \\
6 & $+1,07 p$ & & 8 & $+1,09 p$ & \\
2 & $+1,13 s$ & & 3 & $+1,37 s$ & \\
6 & $+1,75 s$ & & 2 & $+1,94 s$ &
\end{tabular}

\begin{tabular}{l|l|l||l|l|l}
\hline \multicolumn{3}{c||}{$\lambda=3764,6$} & \multicolumn{3}{c}{$\lambda=3459,1$} \\
\hline$i$ & $\Delta \lambda / \lambda^{2}$ & $A$ & $i$ & $\Delta \lambda / \lambda^{2}$ & $A$ \\
\hline \hline \multirow{2}{*}{2} & \multirow{2}{*}{$1,82 s$} & $7 \times, 27$ & $\mathbf{3}$ & $-2,20 s$ & $22 \times, 10$ \\
3 & $1,35 s$ & 5 & 4 & $-1,51 s$ & 15 \\
8 & $1,08 p$ & 4 & 8 & $-1,17 p$ & 12 \\
2 &, $86 s$ & 3 & 2 &,$- 73 s$ & 7 \\
$1+$ &, $86 s$ & & 3 &,$+ 68 s$ & 7 \\
8 & $1,08 p$ & & 8 & $+1,17 p$ & 12 \\
3 & $1,35 s$ & & 4 & $+1,54 s$ & 15 \\
$1+$ & $1,89 s$ & & $1+2,28 s$ & 23
\end{tabular}

4027,4 und 3764,6 sind wahrscheinlich Duplikate. 
Die folgenden sieben Linien haben je sieben Komponenten.

\begin{tabular}{r|c|c||r|c|l}
\hline \multicolumn{3}{c}{$\lambda=4457,71$} & \multicolumn{3}{c}{$\lambda=4258,31$} \\
\hline$i$ & $\Delta \lambda / \lambda^{2}$ & $A$ & $i$ & $\Delta \lambda / \lambda^{2}$ & $A$ \\
\hline \hline 2 & $-2,31 s$ & & 4 & $-1,53 s$ & $6+, 26$ \\
10 & $-1,46 s$ & & 6 & $-1,37 p$ & $5(?)$ \\
10 & $-1,33 p$ & & 2 & $-[, 82] p$ & 3 \\
2 & $0 p$ & 1 & 10 & $0 s$ & 0 \\
10 & $+1,46 s$ & & 2 & $+[, 82] p$ & $8 \times, 10$ \\
10 & $+1,50 p$ & & 6 & $+1,37 p$ & 14 \\
2 & $+2,31 s$ & & 4 & $+1,48 s$ & 15
\end{tabular}

\begin{tabular}{c|c|l||l|l|l}
\hline \multicolumn{3}{c||}{$\lambda=4171,65$} & \multicolumn{3}{c}{$\lambda=4093,32$} \\
\hline$i$ & $\Delta \lambda / \lambda^{2}$ & $A$ & $i$ & $\Delta \lambda / \lambda^{2}$ & \multicolumn{1}{c}{$A$} \\
\hline \hline 8 & $-1,48 s$ & $6 \times, 25$ & $1+$ & $-1,77 s$ & $6 \times, 355(?)$ \\
8 & $-1,26 p$ & 5 & 2 &,$- 90 p$ & 3 \\
6 &,$- 26 s$ & 1 & 2 &,$- 67 s$ & $2(?)$ \\
6 & $0 p$ & $02)$ & 3 & $0 p$ & 0 \\
6 &,$+ 26 s$ & & 3 &,$+ 66 s$ & $7 \times, 10$ \\
4 & $+1,24 p$ & & 2 &,$+ 93 p$ & 9 \\
3 & $+1,50 s$ & & $1+$ & $+1,78 s$ & 18 \\
2 & {$[+3,02 s]$} & $12(?)(\alpha)$ & & &
\end{tabular}

\begin{tabular}{|c|c|c|c|c|c|c|c|c|}
\hline \multicolumn{3}{|c|}{$\lambda=4068,9$} & \multicolumn{3}{|c|}{$\lambda=4055,2$} & \multicolumn{3}{|c|}{$\lambda=3368,01$} \\
\hline$i$ & $\Delta \lambda / \lambda^{2}$ & $A$ & $i$ & $\Delta \lambda / \lambda^{2}$ & $A$ & $i$ & $\Delta \lambda / \lambda^{2}$ & $A$ \\
\hline 2 & $-1,55 s$ & & 2 & $-1,11 s$ & $2 \times, 53$ & 1 & $-1,71 \mathrm{~s}$ & $4 \times, 43$ \\
\hline 2 & $-1,16 p$ & & 6 &,$- 56 p$ & 1 & 8 & $-1,25 p$ & 3 \\
\hline 2 &,$- 49 s$ & & 8 &,$- 53 s$ & 1 & 2 &,$- 88 s$ & 2 \\
\hline 6 & $0 p$ & 3) & 8 & $0 p$ & 0 & 3 & $0 p$ & 0 \\
\hline 2 &,$+ 49 s$ & & 4 &,$+ 49 p$ & $\left.{ }^{8}\right)$ & 2 &,$+ 88 \mathrm{~s}$ & \\
\hline 2 &,$+ 83 p$ & & 8 &,$+ 53 \mathrm{~s}$ & & 8 & $+1,25 p$ & \\
\hline 2 & $+1,55 s$ & & 2 & $+1,11 \mathrm{~s}$ & & $1+$ & $+1,68 \mathrm{~s}$ & \\
\hline
\end{tabular}

1) Die schwache, mittlere parallele Komponente ist unsymmetrisch.

2) Die Linie ist unsymmetrisch in den Intensitäten, mit einer möglichen Extrakomponente nach der violetten Seite. Dies kann eine fremde Linie sein. Sie erscheint nicht auf den Platten mit schwächerem Felde.

3) Das äuBere, schwache parallele Paar von Komponenten ist nicht symmetrisch. 
Die folgenden 16 Linien haben je sechs Komponenten.

\begin{tabular}{c|c|c||c|c|c}
\hline \multicolumn{3}{c||}{$\lambda=4590,81$} & \multicolumn{3}{c}{$\lambda=4438,23$} \\
\hline$i$ & $\Delta \lambda / \lambda^{2}$ & $A$ & $i$ & $\Delta \lambda / \lambda^{2}$ & $A$ \\
\hline \hline 2 & $-1,74 s$ & $3 \times, 55$ & 2 & $-1,57 s$ & $3 \times, 55$ \\
6 & $-1,11 p$ & 2 & 6 & $-1,07 p$ & 2 \\
2 &,$- 55 s$ & 1 & 2 &,$- 57 s$ & 1 \\
2 &,$+ 55 s$ & & 2 &,$+ 55 s$ & $6(?) \times, 10$ \\
5 & $+1,11 p$ & & 6 & $+1,07 p$ & 11 \\
2 & $+1,67 s$ & & 2 & $+1,60 s$ & 16
\end{tabular}

\begin{tabular}{r|l|l||r|c|c}
\hline \multicolumn{3}{c||}{$\lambda=4431,70$} & \multicolumn{3}{c}{$\lambda=4403,67$} \\
\hline$i$ & $\Delta \lambda / \lambda^{2}$ & $A$ & $i$ & $\Delta \lambda / \lambda^{2}$ & $A$ \\
\hline \hline \multirow{2}{*}{6} & $-2,73 s$ & $5 \times, 55$ & 5 & $-2,11 s$ & $8 \times, 26$ \\
6 & $-1,67 s$ & 3 & 15 &,$- 76 p$ & 3 \\
12 & $-1,12 p$ & 2 & 12 &,$- 48 s$ & 2 \\
12 & $+1,12 p$ & & 12 & $+; 48 s$ & \\
6 & $+1,67 s$ & & 15 &,$+ 76 p$ & \\
6 & $+2,74 s$ & & 5 & $+2,11 s$ &
\end{tabular}

\begin{tabular}{|c|c|c|c|c|c|}
\hline \multicolumn{3}{|c|}{$\lambda=4110,29$} & \multicolumn{3}{|c|}{$\lambda=4040,49$} \\
\hline$i$ & $\Delta \lambda / \lambda^{2}$ & $A$ & $i$ & $\Delta \lambda / \lambda^{2}$ & $A$ \\
\hline 6 & $-1,54 s$ & $4 \times, 39$ & 2 & $-2,08 s$ & $8 \times, 26$ \\
\hline 8 & $-1,17 p$ & 3 & 10 &,$- 77 p$ & 3 \\
\hline $1+$ &,$- 77 \mathrm{~s}$ & 2 & 8 &,$- 51 \mathrm{~s}$ & 2 \\
\hline $1+$ &,$+ 77 \mathrm{~s}$ & & 8 & $+51 s$ & \\
\hline 8 & $+1,17 p$ & & 10 &,$+ 77 p$ & \\
\hline 6 & $+1,54 s$ & & 2 & $+2,12 \mathrm{~s}$ & \\
\hline
\end{tabular}

\begin{tabular}{r|l|l||l|l|l}
\hline \hline \multicolumn{3}{c||}{$\lambda=3554,31$} & \multicolumn{3}{c}{$\lambda=3507,80$} \\
\hline$i$ & $\Delta \lambda / \lambda^{2}$ & \multicolumn{1}{c||}{$A$} & $i$ & $\Delta \lambda / \lambda^{2}$ & $A$ \\
\hline \hline 2 & $-2,67 s$ & $6(?) \times, 43$ & 6 & $-2,78 s$ & \\
6 & $-1,72 s$ & 4 & $1+$ & $-[1,05] s$ & \\
10 &,$- 42 p$ & 1 & 5 &,$- 90 p$ & \\
10 &,$+ 42 p$ & $4 \times, 10$ & 5 &,$+ 90 p$ & \\
6 & $+1,72 s$ & 17 & $1+$ & $+1,08] s$ & \\
2 & $+2,67 s$ & 27 & 6 & $+2,75 s$ &
\end{tabular}


Zerlegung der Spektrallinien von Barium usw.

\begin{tabular}{l|l|l||r|r|r}
\hline \multicolumn{3}{c||}{$\lambda=3498,00$} & \multicolumn{3}{c}{$\lambda=3483,70$} \\
\hline$i$ & \multicolumn{1}{c||}{$\Delta \lambda / \lambda^{2}$} & \multicolumn{1}{c||}{$A$} & $i$ & \multicolumn{1}{c}{$\Delta \lambda / \lambda^{2}$} & $A$ \\
\hline \hline $1+$ & $-2,51 s$ & $5 \times, 50$ & 4 & $-1,82 s$ & $5 \times, 36$ \\
6 & $-1,48 s$ & 3 & 16 & $-1,08 s$ & 3 \\
3 &,$- 44 p$ & 1 & 20 &,$- 38 p$ & 1 \\
2 &,$+ 44 p$ & & 20 &,$+ 38 p$ & \\
6 & $+1,48 s$ & & 16 & $+1,08 s$ & \\
$1+$ & $+2,45 s$ & & 4 & $+1,80 p$ &
\end{tabular}

\begin{tabular}{c|l|l||l|l|l}
\hline \multicolumn{3}{c||}{$\lambda=3482,96$} & \multicolumn{3}{c}{$\lambda=3396,51$} \\
\hline$i$ & $\Delta \lambda / \lambda^{2}$ & $A$ & $i$ & $\Delta \lambda / \lambda^{2}$ & $A$ \\
\hline \hline \multirow{2}{*}{4} & $-1,10 s$ & $3 \times, 36$ & 2 & $-1,06 p$ & $3 \times, 34$ \\
4 &,$- 72 p$ & 2 & 6 &,$- 67 s$ & 2 \\
4 &,$- 70 s$ & 2 & 6 &,$- 33 p$ & 1 \\
4 &,$+ 70 s$ & & 6 &,$+ 33 p$ & \\
6 &,$+ 72 p$ & & 6 &,$+ 67 s$ & \\
4 & $+1,10 s$ & & 2 &,$+ 95 p$ & \\
\hline
\end{tabular}

\begin{tabular}{|c|c|c|c|c|c|}
\hline \multicolumn{3}{|c|}{$\lambda=3323,21$} & \multicolumn{3}{|c|}{$\lambda=3318,70$} \\
\hline$i$ & $\Delta \lambda / \lambda^{2}$ & $A$ & $i$ & $\Delta \lambda / \lambda^{2}$ & $A$ \\
\hline 8 & $-1,91 \mathrm{~s}$ & $5 \times, 38$ & - & $-[1,53] s$ & \\
\hline 2 & $-[1,15] s$ & 3 & $1+$ & $-[, 72] s$ & \\
\hline 12 & $-\quad, 41 p$ & 1 & 5 & $-\quad, 69 p$ & \\
\hline 12 &,$+ 41 p$ & & 5 &,$+ 69 p$ & \\
\hline 2 & $+[1,15] s$ & & $1+$ & $+[, 74] s$ & \\
\hline 8 & $+1,91 s$ & & 5 & $+1,45 s$ & \\
\hline \multicolumn{3}{|c|}{$\lambda=3313,89$} & \multicolumn{3}{|c|}{$\lambda=3155,90$} \\
\hline$i$ & $\Delta \lambda / \lambda^{2}$ & $A$ & $i$ & $\Delta \lambda / \lambda^{2}$ & $A$ \\
\hline $1+$ & $-[2,39] s$ & $6 \times, 40$ & 2 & $-2,10 s$ & $8 \times, 26$ \\
\hline 8 & $-1,69 \mathrm{~s}$ & 4 & 6 &,$- 77 p$ & 3 \\
\hline 6 & $-\quad, 40 p$ & 1 & 8 &,$- 51 s$ & 2 \\
\hline 6 & $+\quad, 40 p$ & $4 \times, 10$ & 8 &,$+ 51 \mathrm{~s}$ & \\
\hline 8 & $+1,68 s$ & 17 & 6 &,$+ 77 p$ & \\
\hline $1+$ & $+[2,39] s$ & 24 & 2 & $+2,10 s$ & \\
\hline
\end{tabular}


Die elf folgenden Linien haben je fünf Komponenten.

\begin{tabular}{|c|c|c|c|c|c|c|c|c|}
\hline \multicolumn{3}{|c|}{$\lambda=5350,5$} & \multicolumn{3}{|c|}{$\lambda=4236,23$} & \multicolumn{3}{|c|}{$\lambda=4187,30$} \\
\hline$i$ & $\Delta \lambda / \lambda^{2}$ & $A$ & $i$ & $\Delta \lambda / \lambda^{2}$ & $A$ & $i$ & $\Delta \lambda / \lambda^{2}$ & $A$ \\
\hline 8 & $-1,42 \mathrm{~s}$ & $3 \times, 48$ & 8 & $-1,47 \mathrm{~s}$ & & 5 & $-2,92 s$ & $9 \times, 32$ \\
\hline 4 &,$- 49 p$ & 1 & 2 &,$- 66 p$ & & 4 & $-1,26 p$ & 4 \\
\hline 2 & $0 s$ & 0 & 4 & $0 p$ & & 8 & $0 p$ & 0 \\
\hline 3 &,$+ 49 p$ & & 2 &,$+ 66 p$ & & 4 & $+1,24 p$ & \\
\hline 4 & $+1,44 s$ & & 8 & $\begin{array}{r}+1,47 s \\
{[2,20] ? s}\end{array}$ & & 5 & $+2,92 s$ & \\
\hline
\end{tabular}

\begin{tabular}{|c|c|c|c|c|c|c|c|c|}
\hline \multicolumn{3}{|c|}{$\lambda=4061,70$} & \multicolumn{3}{|c|}{$\lambda=4046,30$} & \multicolumn{3}{|c|}{$\lambda=3501,50$} \\
\hline$i$ & $\Delta \lambda / \lambda^{2}$ & $A$ & $i$ & $\Delta \lambda / \lambda^{2}$ & $A$ & $i$ & $\Delta \lambda / \lambda^{2}$ & $\bar{A}$ \\
\hline 10 & $-1,79 s$ & $7 \times, 26$ & 2 & $-1,65 s$ & $2 \times, 83$ & 6 & $-2,00 s$ & $3 \times, 67$ \\
\hline 4 &,$- 52 p$ & 2 & 4 &,$- 84 p$ & 1 & 4 & $-[, 70] x$ & 1 \\
\hline 6 & $0 p$ & 0 & 8 & $0 s$ & & 2 & $0 s$ & 0 \\
\hline 3 &,$+ 51 p$ & & 4 & $-p$ & & 2 & $+[, 70] p$ & $7 \times, 10$ \\
\hline 10 & $+1,79 p$ & & 2 & $+1,65 \mathrm{~s}$ & & 4 & $+2,00 s$ & 20 \\
\hline
\end{tabular}

\begin{tabular}{|c|c|c|c|c|c|c|c|c|}
\hline \multicolumn{3}{|c|}{$\lambda=3471,31$} & \multicolumn{3}{|c|}{$\lambda=3432,59$} & \multicolumn{3}{|c|}{$\lambda=3376,42$} \\
\hline$i$ & $\Delta \lambda / \lambda^{2}$ & $A$ & $i$ & $\Delta \lambda / \lambda^{2}$ & $A$ & $i$ & $\Delta \lambda / \lambda^{2}$ & $A$ \\
\hline 6 &,$- 87 s$ & $9 \times, 10$ & 2 & $-1,71 \mathrm{~s}$ & & 2 & $-1,10 p$ & $3 \times, 37$ \\
\hline 12 &,$- 30 p$ & 3 & 8 &,$- 94 p$ & & 2 &,$- 38 p$ & 1 \\
\hline 6 &,$+ 30 p$ & 3 & 15 & $0 s$ & & 10 & $0 s$ & 0 \\
\hline 4 &,$+ 49 s$ & 5 & 8 &,$+ 94 p$ & & 2 &,$+ 38 p$ & \\
\hline 2 & $+1,20 \mathrm{~s}$ & 12 & 2 & $+1,71 \mathrm{~s}$ & & $1+$ & $+1,17 p$ & \\
\hline
\end{tabular}

\begin{tabular}{r|c|l||l|l|l}
\hline \multicolumn{3}{c}{$\lambda=3271,30$} & \multicolumn{3}{c}{$\lambda=3099,42$} \\
\hline$i$ & $\Delta \lambda / \lambda^{2}$ & $A$ & $i$ & $\Delta \lambda / \lambda^{2}$ & $A$ \\
\hline \hline 2 & $-[1,38] s$ & & $1+$ & - & \\
8 &,$- 63 p$ & & 5 & $1,31 p$ & \\
12 & $0 s$ & & 3 & $0 p$ & \\
8 &,$+ 63 p$ & & 5 & $1,31 p$ & \\
2 & $+[1,38] s$ & & $1+$ & - &
\end{tabular}

Linie 5350,5 ist unsymmetrisch in der Intensität. Die Linie 3471,31 ist unsymmetrisch sowohl in der Intensität als auch in der Zerlegung. Duplikate sind nicht vorhanden. 
Die folgenden 89 Linien sind in vier Komponenten zerlegt.

\begin{tabular}{|c|c|c|c|c|c|c|c|}
\hline$\lambda$ & $i$ & $\Delta \lambda / \lambda^{2}$ & $A$ & $\lambda$ & $i$ & $\Delta \lambda / \lambda^{2}$ & $A$ \\
\hline 4582,50 & $\begin{array}{l}3 \\
2\end{array}$ & $\begin{array}{r}\mp 1,02 s \\
, 52 p\end{array}$ & $\begin{array}{l}2 \times, 51 \\
1\end{array}$ & 4017,16 & $\begin{array}{l}6 \\
8\end{array}$ & $\begin{array}{r}1,13 \\
, 43\end{array}$ & $\begin{array}{l}3 \times, 42 \\
1\end{array}$ \\
\hline 4456,50 & $\begin{array}{l}30 \\
30\end{array}$ & $\begin{array}{l}, 83 \\
, 56\end{array}$ & $\begin{array}{l}3 \times, 28 \\
2\end{array}$ & 4004,51 & $\begin{array}{l}2 \\
5\end{array}$ & $\begin{array}{r}1,00 \\
, 79\end{array}$ & $\begin{array}{l}5 \times, 20 \\
4\end{array}$ \\
\hline 4455,64 & $\begin{array}{l}2 \\
2\end{array}$ & $\begin{array}{r}1,20 \\
, 84\end{array}$ & $\begin{array}{l}3 \times, 42 \\
2\end{array}$ & 4003,28 & $\begin{array}{l}8,10 \\
6,4\end{array}$ & $\begin{array}{r}2,05 \\
, 29\end{array}$ & $\begin{array}{l}7 \times, 29 \\
1\end{array}$ \\
\hline 4455,08 & $\begin{array}{l}12 \\
15\end{array}$ & $\begin{array}{r}1,21 \\
, 46\end{array}$ & $\begin{array}{l}3 \times, 42 \\
1\end{array}$ & 3999,18 & $\begin{array}{l}100 \\
100\end{array}$ & $\begin{array}{r}1,17 \\
, 85\end{array}$ & $\begin{array}{l}3 \times, 42(?) \\
2\end{array}$ \\
\hline 4429,28 & $\begin{array}{c}3 \\
5,3\end{array}$ & $\begin{array}{r}1,58 \\
, 49\end{array}$ & $\begin{array}{l}3 \times, 51 \\
1\end{array}$ & 3973,68 & $\begin{array}{l}20 \\
15\end{array}$ & $\begin{array}{r}1,14 \\
, 57\end{array}$ & $\begin{array}{l}2 \times, 57 \\
1\end{array}$ \\
\hline 4420,70 & $\begin{array}{l}15 \\
15\end{array}$ & $\begin{array}{r}1,82 \\
, 71\end{array}$ & & 3934,99 & $\begin{array}{l}20 \\
15\end{array}$ & $\begin{array}{r}1,14 \\
, 57\end{array}$ & $\begin{array}{l}2 \times, 57 \\
1\end{array}$ \\
\hline $\mathbf{4 4 1 4 , 8 0}$ & $\begin{array}{l}20 \\
20\end{array}$ & $\begin{array}{r}1,58 \\
, 63\end{array}$ & $\begin{array}{l}5 \times, 32 \\
2\end{array}$ & 3916,16 & $\begin{array}{l}25 \\
20\end{array}$ & $\begin{array}{l}, 85 \\
, 24\end{array}$ & $\begin{array}{l}4 \times, 22 \\
1\end{array}$ \\
\hline 4413,28 & $\begin{array}{l}12 \\
15\end{array}$ & $\begin{array}{r}1,76 \\
, 53\end{array}$ & & 3894,00 & $\begin{array}{l}5,3 \\
2,4\end{array}$ & {$\left[\begin{array}{l}, 89] \\
{[, 67]}\end{array}\right.$} & $\begin{array}{l}4 \times, 22 \\
3\end{array}$ \\
\hline 4394,73 & $\overline{7,5}$ & $\overline{, 60}$ & & 3891,61 & 25 & $\begin{array}{r}1,28 \\
, 70\end{array}$ & $13 \times, 10$ \\
\hline 4349,10 & $\begin{array}{l}2 \\
2\end{array}$ & $\begin{array}{c}{[1,23]} \\
, 77\end{array}$ & $\begin{array}{l}3 \times, 40 \\
2\end{array}$ & 3890,58 & $\begin{array}{l}30 \\
30\end{array}$ & $\begin{array}{r}1,29 \\
, 29\end{array}$ & 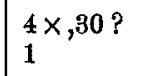 \\
\hline 4312,47 & $\begin{array}{l}4 \\
4\end{array}$ & $\begin{array}{r}1,27 \\
, 41\end{array}$ & $\begin{array}{l}3 \times, 42 \\
1\end{array}$ & 3817,80 & $\begin{array}{c}30,20 \\
20\end{array}$ & $\begin{array}{l}, 81 \\
, 35\end{array}$ & \\
\hline 4309,20 & $\begin{array}{l}8 \\
8\end{array}$ & $\begin{array}{r}1,25 \\
, 85\end{array}$ & $\begin{array}{l}3 \times, 42 \\
2\end{array}$ & 3782,45 & $\begin{array}{c}3,5 \\
7\end{array}$ & $\begin{array}{c}{[, 72]} \\
, 73\end{array}$ & \\
\hline 4256,66 & 6,4 & $\begin{array}{r}1,50 \\
, 27\end{array}$ & $\begin{array}{l}6 \times, 25 \\
1\end{array}$ & 3674,98 & $\begin{array}{l}50 \\
50\end{array}$ & $\begin{array}{l}, 63 \\
, 42\end{array}$ & $\begin{array}{l}3 \times, 21 \\
2\end{array}$ \\
\hline 4224,42 & $\overline{5}$ & $\overline{1,08}$ & & 3663,81 & $\begin{array}{c}20 \\
15,12\end{array}$ & $\begin{array}{r}1,27 \\
, 42\end{array}$ & $\begin{array}{l}3 \times, 42 \\
1\end{array}$ \\
\hline 4199,30 & $\begin{array}{l}15 \\
12\end{array}$ & $\begin{array}{r}1,47 \\
, 46\end{array}$ & $\begin{array}{l}3 \times, 48 \\
1\end{array}$ & 3650,90 & $\begin{array}{r}6 \\
5\end{array}$ & $\begin{array}{r}1,49 \\
, 76\end{array}$ & $2 \times, 75$ \\
\hline 4077,27 & $\overline{5}$ & $\overline{, 38}$ & & 3634,33 & $\begin{array}{c}10 \\
10,6\end{array}$ & $\begin{array}{r}1,36 \\
, 35\end{array}$ & $\begin{array}{l}4 \times, 34 \\
1\end{array}$ \\
\hline 4071,30 & $\begin{array}{r}8 \\
10\end{array}$ & $\begin{array}{r}1,68 \\
, 58\end{array}$ & & 3629,29 & $\begin{array}{l}2 \\
2\end{array}$ & $\begin{array}{r}1,09 \\
, 71\end{array}$ & \\
\hline 4043,77 & $\begin{array}{l}15 \\
15\end{array}$ & $\begin{array}{r}1,48 \\
, 54\end{array}$ & $\begin{array}{l}3 \times, 50 \\
1\end{array}$ & 3624,10 & $\begin{array}{c}25 \\
25,20\end{array}$ & $\begin{array}{r}1,16 \\
, 33\end{array}$ & \\
\hline 4036,10 & $\begin{array}{l}20 \\
20\end{array}$ & $\begin{array}{r}1,30 \\
, 67\end{array}$ & $\begin{array}{l}4 \times, 33 \\
2\end{array}$ & 3613,30 & $\begin{array}{l}40 \\
30\end{array}$ & $\begin{array}{c}{[1,03]} \\
, 95\end{array}$ & \\
\hline 4034,30 & $\begin{array}{l}6 \\
4\end{array}$ & $\begin{array}{r}1,33 \\
, 32\end{array}$ & $4 \times, 33$ & 3612,61 & $\begin{array}{l}3 \\
3\end{array}$ & $\begin{array}{l}, 59 \\
, 41\end{array}$ & \\
\hline 4031,57 & $\begin{array}{l}\mathbf{2} \\
\mathbf{3}\end{array}$ & $\begin{array}{r}1,66 \\
, 41\end{array}$ & $\begin{array}{l}4 \times, 42 \\
1\end{array}$ & 3588,15 & $\begin{array}{l}20 \\
20\end{array}$ & $\begin{array}{l}, 58 \\
, 29\end{array}$ & $\begin{array}{l}2 \times, 29 \\
1\end{array}$ \\
\hline 4025,16 & $\begin{array}{l}5 \\
25 \\
25\end{array}$ & $\begin{array}{c}{[1,39]} \\
, 73\end{array}$ & & 3578,40 & 20 & $\mp 2,01 s$ & $\begin{array}{l}7 \times, 29 \\
1\end{array}$ \\
\hline 4024,20 & $\begin{array}{l}\mathbf{2 5} \\
\mathbf{2 0}\end{array}$ & $\begin{array}{r} \pm 1,54 s \\
, 55 p\end{array}$ & $\begin{array}{l}3 \times, 51 \\
1\end{array}$ & 3569,03 & $\begin{array}{l}2 \\
2\end{array}$ & {$[\overline{-47}$} & \\
\hline
\end{tabular}




\begin{tabular}{|c|c|c|c|c|c|c|c|}
\hline$\lambda$ & $i$ & $A \lambda / \lambda^{2}$ & $A$ & $\lambda$ & $i$ & $\Delta \lambda / \lambda^{2}$ & $A$ \\
\hline 3568,32 & $\begin{array}{l}6 \\
6\end{array}$ & $\begin{array}{r}1,17 \\
, 90\end{array}$ & $\begin{array}{l}4 \times, 30 \\
3\end{array}$ & 3357,42 & $\begin{array}{l}25,20 \\
25,20\end{array}$ & $\begin{array}{l}, 59 \\
, 40\end{array}$ & $\begin{array}{l}3 \times, 20 \\
2\end{array}$ \\
\hline 3552,20 & $\begin{array}{l}50 \\
50\end{array}$ & $\begin{array}{r}1,27 \\
, 77\end{array}$ & $\begin{array}{l}3 \times, 42 \\
2(?)\end{array}$ & 3338,07 & $\begin{array}{l}4 \\
2\end{array}$ & $\begin{array}{l}1,98 \\
1,17\end{array}$ & \\
\hline 3530,17 & $\begin{array}{c}6 \\
5,4\end{array}$ & 1,21 & $\begin{array}{l}3 \times, 42 \\
1\end{array}$ & 3310,10 & $\begin{array}{l}4 \\
4\end{array}$ & $\overline{1,45}$ & \\
\hline 3514,79 & $\begin{array}{l}4 \\
4\end{array}$ & $\begin{array}{r}1,23 \\
, 84\end{array}$ & $\begin{array}{l}3 \times, 42 \\
2\end{array}$ & 3296,59 & $\begin{array}{l}6 \\
4\end{array}$ & $\begin{array}{l}1,49 \\
{[, 47]}\end{array}$ & $\begin{array}{l}3 \times, 50 \\
1\end{array}$ \\
\hline 3505,67 & $\begin{array}{l}60 \\
60\end{array}$ & $\begin{array}{r}1,67 \\
, 41\end{array}$ & $\begin{array}{l}4 \times, 42 \\
1\end{array}$ & 3287,46 & $\begin{array}{l}2 \\
2\end{array}$ & $\overline{54}$ & \\
\hline 3485,48 & $\begin{array}{r}10 \\
8\end{array}$ & $\begin{array}{l}1,74 \\
1,11\end{array}$ & & 3284,89 & $\begin{array}{l}20 \\
20\end{array}$ & ,65 & $\begin{array}{l}2 \times, 33 \\
1\end{array}$ \\
\hline 3480,59 & $\begin{array}{l}15 \\
15\end{array}$ & $\begin{array}{r}1,57 \\
, 98\end{array}$ & $\begin{array}{l}3 \times, 51(?) \\
2\end{array}$ & 3274,14 & $\begin{array}{l}4 \\
4\end{array}$ & $\begin{array}{r}1,46 \\
, 78\end{array}$ & $\begin{array}{l}2 \times, 75 \\
1\end{array}$ \\
\hline 3478,98 & $\begin{array}{l}2 \\
6\end{array}$ & $\begin{array}{c}{[, 87]} \\
, 50\end{array}$ & & 3264,96 & $\begin{array}{l}4 \\
4\end{array}$ & $\begin{array}{l}1,46 s \\
{[, 51] p}\end{array}$ & $\begin{array}{l}3 \times, 50 \\
1\end{array}$ \\
\hline 3470,10 & $\begin{array}{l}2 \\
4\end{array}$ & $\begin{array}{c}{[1,59]} \\
1,33\end{array}$ & $\begin{array}{l}6 \times, 27 \\
5\end{array}$ & 3229,00 & 15,12 & $\begin{array}{r}1,36 \\
, 46\end{array}$ & $\begin{array}{l}3 \times, 45 \\
1\end{array}$ \\
\hline 3460,10 & $\begin{array}{c}2,3 \\
3\end{array}$ & $\begin{array}{c}{[1,22]} \\
, 72\end{array}$ & 2 & 3213,01 & $\begin{array}{l}4 \\
4\end{array}$ & $\begin{array}{r}1,10 \\
, 50\end{array}$ & $\begin{array}{l}2 \times, 53(?) \\
1\end{array}$ \\
\hline 3454,71 & $\begin{array}{l}3 \\
4\end{array}$ & $\begin{array}{r}, 61 \\
1,18\end{array}$ & $\begin{array}{l}1 \times, 60 \\
2\end{array}$ & 3182,15 & $\overline{5}$ & $\overline{67}$ & \\
\hline 3440,54 & $\begin{array}{l}5 \\
6\end{array}$ & $\begin{array}{l}0,+ \\
1,11\end{array}$ & $p>s$ & 3181,79 & $\begin{array}{l}8 \\
8\end{array}$ & $\begin{array}{l}1,56 \\
1,45\end{array}$ & \\
\hline 3437,30 & $\begin{array}{l}25 \\
25\end{array}$ & $\begin{array}{r}1,42 \\
, 72\end{array}$ & $\begin{array}{l}2 \times, 71 \\
1\end{array}$ & 3178,30 & $\begin{array}{l}8 \\
8\end{array}$ & $\begin{array}{r}1,65 \\
, 87\end{array}$ & $\begin{array}{l}4 \times, 42 \\
2\end{array}$ \\
\hline 3434,08 & $\begin{array}{r}5 \\
10\end{array}$ & $\begin{array}{l}1,58 \\
1,19\end{array}$ & $\begin{array}{l}4 \times, 39 \\
3\end{array}$ & 3166,48 & $\overline{6}$ & $\overline{61}$ & \\
\hline 3424,97 & $\begin{array}{c}4 \\
10,8\end{array}$ & $\begin{array}{l}, 93 \\
, 41\end{array}$ & $\begin{array}{l}2 \times, 42(?) \\
1\end{array}$ & 3165,68 & $\begin{array}{l}4 \\
4\end{array}$ & $\begin{array}{r}1,60 \\
, 49\end{array}$ & \\
\hline 3413,55 & $\begin{array}{l}2 \\
4\end{array}$ & $\begin{array}{c}{[1,55]} \\
1,43\end{array}$ & $\begin{array}{l}13 \times, 12 \\
12\end{array}$ & 3164,54 & $\begin{array}{r}12 \\
6\end{array}$ & $\begin{array}{r}1,09 \\
, 40\end{array}$ & $\left.\right|_{4} ^{11 \times, 10}$ \\
\hline 3394,96 & $\begin{array}{l}4 \\
4\end{array}$ & $\begin{array}{r}1,76 \\
, 94\end{array}$ & & 3157,19 & $\begin{array}{l}6 \\
3\end{array}$ & $\begin{array}{r}1,16 \\
, 81\end{array}$ & $\begin{array}{l}3 \times, 42(?) \\
2\end{array}$ \\
\hline 3378,47 & $\begin{array}{l}4 \\
4\end{array}$ & $\overline{1,43}$ & & 3111,09 & $\begin{array}{l}4 \\
6\end{array}$ & $\begin{array}{r}1,46 \\
, 55\end{array}$ & \\
\hline 3377,61 & $\begin{array}{c}8 \\
6,4\end{array}$ & $\begin{array}{r}1,45 \\
, 61\end{array}$ & & 3095,29 & $\begin{array}{l}8 \\
8\end{array}$ & $\begin{array}{r}1,25 \\
, 76\end{array}$ & $\begin{array}{l}5 \times, 25 \\
3\end{array}$ \\
\hline $\begin{array}{l}3369,42 \\
3364,00\end{array}$ & $\begin{array}{l}4 \\
4 \\
8 \\
8\end{array}$ & $\begin{array}{r}1,64 \\
, 44 \\
\mp 1,33 s \\
, 84 p\end{array}$ & $\begin{array}{l}4 \times, 42 \\
1 \\
3 \times, 42 \\
2\end{array}$ & 3031,04 & $\begin{array}{l}3 \\
2\end{array}$ & $\overline{6}, \overline{6}$ & \\
\hline
\end{tabular}

Eine auffallende Verwandtschaft zwischen den Komponenten einer Linie scheint zwischen einer großen Zahl dieser Quadruplette $\mathrm{zu}$ bestehen. Das Intervall von 0,42 wiederholt sich sehr oft bei vielen Linien, aber die Faktoren, mit denen dies Intervall multipliziert werden muB, um die Zerlegungen der Linien zu bekommen, sind ganz verschieden, so daB es wenig Duplikatlinien gibt. Einige von diesen Linien sind offenbar Paare. 
Die folgenden 410 Linien sind Triplette.

\begin{tabular}{|c|c|c|c|c|c|}
\hline$\lambda$ & $i$ & $\Delta \lambda / \lambda^{2}$ & $\lambda$ & $i$ & $\Delta \lambda / \lambda^{2}$ \\
\hline 5665,4 & 2,4 & $\mp 1,25$ & 4645,01 & 6,12 & $\mp, 76$ \\
\hline 36,0 & $1+, 3$ & {$[, 83]$} & 34,20 & 15,30 & 1,09 \\
\hline 5504,7 & 6,12 &, 59 & 29,33 & 6,12 & 1,20 \\
\hline 02,5 & 6,12 &, 99 & 26,62 & 8,15 & 1,21 \\
\hline 5492,2 & 5,10 & ,83 & 14,20 & 6,12 & 1,03 \\
\hline 69,8 & 8,16 &, 56 & 04,59 & 3,6 & 2,34 \\
\hline 65,6 & $1+, 3$ & {$[1,01]$} & 02,80 & 10,20 & 1,27 \\
\hline 53,8 & 6,12 &, 66 & 4584,44 & $1+, 3$ & {$[1,02]$} \\
\hline 46,0 & 6,10 &, 76 & 76,37 & $1+, 3$ &, 71 \\
\hline 00,7 & 8,15 & ,68 & 75,78 & 15,30 &, 91 \\
\hline 5385,6 & 8,15 & 1,24 & 74,78 & 6,12 &, 94 \\
\hline 57,5 & 8,15 &, 66 & 65,68 & 3,6 &, 95 \\
\hline 5191,7 & 10,20 & 1,30 & 54,29 & 8,16 & 1,10 \\
\hline 43,5 & $6,12,8$ &, 62 & 53,25 & 3,6 &, 54 \\
\hline 38,4 & 10,20 & ,62 & 42,49 & 12,25 & 1,59 \\
\hline 14,2 & 2,4 & {$[, 90]$} & 40,19 & 2,6 & {$[1,31]$} \\
\hline 5079,6 & $1+, 3$ & {$[, 62]$} & 36,00 & 10,20 &, 94 \\
\hline 66,9 & 2,5 &, 95 & 23,33 & 3,6 & 1,35 \\
\hline 4839,3 & $2,5,4$ &, 75 & 07,39 & 8,15 & 1,10 \\
\hline 28,5 & $4,6,3$ &, 70 & 4497,27 & 30,60 &, 94 \\
\hline 24,8 & 4,8 & 1,27 & 94,78 & 15,30 & 1,20 \\
\hline 16,1 & 12,24 &, 78 & 85,71 & 2,4 & {$[2,35]$} \\
\hline 10,0 & 4,8 & 1,19 & 82,72 & 4,8 & 1,24 \\
\hline 06,5 & 4,8 & 1,27 & 81,00 & 5,10 & 1,28 \\
\hline 4789,3 & 3,6 &, 95 & 70,80 & 10,20 & 1,08 \\
\hline 85,6 & 6,12 &, 99 & 68,98 & 3,8 & 1,29 \\
\hline 72,1 & 15,30 &, 90 & 67,12 & 6,12 & 1,53 \\
\hline 62,8 & 2,4 & 1,13 & 61,50 & 8,15 & 1,18 \\
\hline 39,6 & 20,4 & 1,04 & 60,57 & 2,4 & 1,27 \\
\hline 19,3 & 8,16 & 1,17 & 50,71 & 5,10 & 2,73 \\
\hline 12,2 & 3,6 & 1,31 & 43,31 & 30,60 & 1,07 \\
\hline 10,3 & 25,50 & 1,18 & 29,55 & $1+, 3$ & {$[1,61]$} \\
\hline 03,3 & 2,4 & {$[1,41]$} & 27,44 & 8,15 & 1,09 \\
\hline 4688,7 & 5,8 & 1,14 & 04,98 & 6,12 & 1,44 \\
\hline 88,0 & 30,60 & 1,32 & 4383,90 & 6,12 & 1,31 \\
\hline 85,5 & 3,8 & 1,11 & 80,12 & 50,100 & 1,15 \\
\hline 83,6 & 8,16 & 1,19 & 73,28 & $1+, 3$ & {$[2,32]$} \\
\hline 67,4 & $1+, 3$ & {$[1,24]$} & 71,27 & 20,40 & 1,18 \\
\hline 62,0 & 3,8 & 1,05 & 66,69 & 15,30 & 1,22 \\
\hline 57,9 & 4,8 & 1,18 & 61,01 & 6,12 & 1,62 \\
\hline
\end{tabular}




\begin{tabular}{|c|c|c|c|c|c|}
\hline$\lambda$ & $i$ & $\Delta \lambda / \lambda^{2}$ & $\lambda$ & $i$ & $\Delta \lambda / \lambda^{2}$ \\
\hline 4360,05 & 50,100 & $\mp 1,26$ & 4218,60 & 4,8 & $\mp 1,86$ \\
\hline 58,95 & 5,10 & 1,32 & 15,95 & 4,8 &, 84 \\
\hline 48,15 & 50,100 & 1,12 & 15,50 & 6,12 & 1,42 \\
\hline 46,70 & 5,10 &, 84 & 12,17 & 25,50 &, 62 \\
\hline 43,59 & $1+, 3$ & {$[1,23]$} & 11,50 & 4,8 & 1,57 \\
\hline 43,23 & 2,4 & 1,06 & 10,87 & 10,20 & 1,57 \\
\hline 41,40 & 5,10 & 1,36 & 09,21 & 50,100 &, 97 \\
\hline 39,80 & 3,6 & 1,86 & 01,69 & 20,50 & 1,69 \\
\hline 37,90 & 8,15 & 1,34 & 419632 & 8,15 & 1,35 \\
\hline 33,59 & 15,30 & 1,24 & 95,00 & 10,20 & 1,84 \\
\hline 24,24 & 8,15 & 1,06 & 94,66 & 2,4 & 1,20 \\
\hline 19,24 & 4,8 & 1,24 & 92,03 & 4,8 & {$[1,14]$} \\
\hline 17,57 & 12,25 & 1,26 & 91,75 & 3,5 &, 77 \\
\hline 09,99 & 4,8 & 1,68 & 87,80 & 20,30 & 1,60 \\
\hline 04,92 & 8,15 & 1,45 & 86,89 & 20,30 & 1,63 \\
\hline 03,12 & 20,40 & 1,67 & 83,51 & 10,20 & 1,77 \\
\hline 02,80 & 2,4 & 1,67 & 80,08 & 25,40 & 1,40 \\
\hline 02,10 & 8,16 &, 88 & 68,90 & 6,10 & 1,28 \\
\hline 4297,00 & 12,25 & 1,50 & 66,60 & 15,30 & 1,50 \\
\hline 95,08 & 25,30 & 1,69 & 61,48 & 30,50 &, 71 \\
\hline 93,41 & 15,30 &, 75 & 53,96 & 8,15 & 2,38 \\
\hline 91,54 & 5,10 & 1,63 & 52,87 & 15,30 & 1,59 \\
\hline 91,38 & 4,8 & 1,62 & 51,23 & 20,50 & 1,39 \\
\hline 86,78 & 6,12 &, 76 & 49,43 & 150,300 & 1,27 \\
\hline 82,53 & 8,15 & 1,82 & 47,53 & 6,10 & 1,20 \\
\hline 77,60 & 6,12 & 1,42 & 40,70 & 5,10 & 1,86 \\
\hline 76,91 & 6,12 & 1,67 & 38,02 & 6,10 & ,33 \\
\hline 74,95 & 6,12 & 1,20 & 35,85 & 10,20 & 1,50 \\
\hline 65,17 & 4,8 & 1,52 & 34,46 & 4,8 & {$[1,66]$} \\
\hline 61,65 & 6,12 & 1,11 & 21,68 & 12,20 & 1,70 \\
\hline 61,42 & 2,5 & {$[, 90]$} & 08,60 & 10,20 & 1,67 \\
\hline 53,76 & 8,15 & 2,14 & 07,75 & 6,10 & ,99 \\
\hline 43,74 & 2,4 & {$[1,44]$} & 4099,50 & 4,6 & {$[1,89]$} \\
\hline 41,98 & 20,40 & 1,27 & 96,80 & 15,30 &, 90 \\
\hline 41,50 & 15,30 & 1,11 & 94,42 & 6,10 & 1,46 \\
\hline 39,58 & 30,20 & 1,49 & 91,00 & 6,10 & ,66 \\
\hline 37,57 & 4,8 & 2,18 & 90,70 & 30,50 &, 91 \\
\hline 36,81 & 6,12 & {$[, 85]$} & 89,98 & 8,15 & 1,00 \\
\hline 34,89 & 8,16 & 1,25 & 87,88 & 5,10 & 1,74 \\
\hline 31,88 & 10,20 & 1,34 & 84,50 & 8,15 & 1,18 \\
\hline 27,98 & 40,60 & 1,51 & 83,29 & 6,12 & 1,58 \\
\hline 22,67 & 4,8 &, 70 & 81,48 & 30,50 & 1,33 \\
\hline
\end{tabular}




\begin{tabular}{|c|c|c|c|c|c|}
\hline$\lambda$ & $i$ & $\Delta \lambda / \lambda^{2}$ & $\lambda$ & $i$ & $\Delta \lambda / \lambda^{2}$ \\
\hline 4078,49 & $6,12,8$ & $\mp 1,66$ & 3916,80 & 6,12 & $\mp 1,42$ \\
\hline 76,70 & 8,15 & 1,26 & 14,59 & 10,20 &, 94 \\
\hline 75,09 & 12,20 & 1,67 & 00,08 & 2,4 & {$[1,65]$} \\
\hline 72,90 & 40,75 & 1,27 & 3897,82 & 5,10 & 1,43 \\
\hline 64,38 & 25,50 & 1,05 & 96,73 & 10,20 & 1,18 \\
\hline 60,78 & 6,10 & 1,03 & 92,19 & 6,12 & 1,21 \\
\hline 58,78 & 4,8 &, 88 & 85,61 & 12,25 & 1,11 \\
\hline 55,90 & 15,25 & 1,32 & 79,21 & 10,20 & 1,35 \\
\hline 54,60 & 2,4 &, 56 & 77,78 & 12,25 & 1,10 \\
\hline 50,52 & 30,50 & 1,37 & 64,57 & 25,50 & 1,32 \\
\hline 48,90 & 30,50 & 1,34 & 64,12 & 30,50 & 1,20 \\
\hline 45,90 & 25,40 &, 61 & 49,48 & 15,25 & 1,35 \\
\hline 42,49 & 8,15 & {$[1,38]$} & 47,22 & 12,20 & 1,28 \\
\hline 41,90 & 10,20 & {$[1,64]$} & 43,30 & 25,50 & 1,05 \\
\hline 30,87 & 2,4 &, 96 & 36,98 & 75,150 & 1,24 \\
\hline 29,88 & 30,50 & 1,77 & 36,18 & 25,50 &, 76 \\
\hline 29,17 & 6,12 & 1,74 & 22,60 & 15,30 &, 85 \\
\hline 24,70 & 20,40 & {$[1,39]$} & 00,91 & 5,10 & 1,44 \\
\hline 18,60 & 15,30 & 1,02 & 3796,71 & 30,50 & 1,80 \\
\hline 12,48 & 8,15 &, 94 & 92,55 & 10,20 & 1,38 \\
\hline 07,80 & 10,20 & 1,08 & 91,60 & 15,25 & 1,52 \\
\hline 05,02 & 6,12 & {$[1,57]$} & 86,80 & 4,8 & 1,34 \\
\hline 3991,31 & 100,150 & 1,08 & 82,99 & 3,6 & {$[, 92]$} \\
\hline 89,65 & 10,15 & 1,33 & 82,45 & 3,6 & {$[, 72]$} \\
\hline 84,90 & 10,20 & 1,57 & 72,89 & 10,20 & 1,55 \\
\hline 81,79 & 20,40 & 1,35 & 66,99 & 50,100 & 1,15 \\
\hline 78,86 & 6,12 & 1,47 & 59,30 & 3,6 & 1,27 \\
\hline 78,35 & 2,4 & {$[1,04]$} & 57,99 & 12,25 & 1,31 \\
\hline 77,60 & 10,20 & 1,06 & 51,85 & 75,150 & 1,02 \\
\hline 75,47 & 12,30 & 1,22 & 50,84 & 10,20 & 1,30 \\
\hline 68,39 & 10,20 & 1,16 & 46,18 & 50,100 & 1,19 \\
\hline 66,80 & 10,20 & 1,69 & 38,32 & 6,10 & 1,47 \\
\hline 61,71 & 12,25 & 1,29 & 37,54 & 4,8 & 1,03 \\
\hline 58,39 & 75,125 &, 98 & 35,15 & 12,20 & 1,52 \\
\hline 56,90 & 3,6 &, 62 & 31,50 & 30,50 &, 97 \\
\hline 41,75 & $10,15,8$ & 1,01 & 29,98 & 8,15 & 1,87 \\
\hline 36,28 & 12,25 & 1,88 & 27,90 & 15,20 & 1,60 \\
\hline 31,55 & 2,4 & 1,66 & 19,02 & 8,12 & 1,59 \\
\hline 29,71 & 30,50 & 1,14 & 14,99 & 30,50 & 1,46 \\
\hline 26,96 & 3,6 & 1,19 & 14,30 & 6,10 & 2,05 \\
\hline 20,35 & 2,4 & {$[1 ; 67]$} & 09,51 & 40,75 & 1,20 \\
\hline 18,25 & $1+, 3$ & {$[, 50]$} & 3698,41 & 75,125 & 1,26 \\
\hline
\end{tabular}




\begin{tabular}{|c|c|c|c|c|c|}
\hline$\lambda$ & $i$ & $\Delta \lambda / \lambda^{2}$ & $\lambda$ & $i$ & $\Delta \lambda / \lambda^{2}$ \\
\hline 3697,70 & 20,40 & $\mp 1,28$ & 3526,00 & $2 \overline{5}, 40$ & $\mp 1,70$ \\
\hline 79,80 & $1+, 3$ & {$[1,37]$} & 21,01 & 12,20 & 1,93 \\
\hline 79,10 & 15,25 & 1,24 & 19,72 & 25,40 & 1,19 \\
\hline 72,81 & 3,5 & 2,85 & 10,61 & 8,12 & 1,72 \\
\hline 71,49 & 25,40 & ,99 & 09,48 & 20,30 & 1,23 \\
\hline 68,69 & 15,25 & 1,73 & 06,23 & 10,15 & 1,89 \\
\hline 67,28 & 6,12 & 1,32 & 05,88 & 75,125 & {$[1,44]$} \\
\hline 62,32 & 10,20 &, 95 & 00,33 & 5,10 & 1,04 \\
\hline 61,10 & 3,5 &, 57 & $\mathbf{3 4 9 9 , 7 8}$ & 20,30 & 1,64 \\
\hline 55,72 & 8,15 & 1,74 & 96,40 & 60,100 & 1,10 \\
\hline 53,61 & 2,4 & 1,17 & 83,17 & 3,5 & {$[1,38]$} \\
\hline 51,65 & 2,4 & {$[1,44]$} & 81,36 & 50,100 & 1,19 \\
\hline 36,69 & 15,25 & 1,69 & 79,58 & 10,15 & {$[1,07]$} \\
\hline 33,70 & 12,20 & 1,29 & 78,68 & 8,12 & 1,63 \\
\hline 30,30 & 20,40 & 1,89 & 78,45 & 6,10 & 1,74 \\
\hline 19,22 & 6,12 & 83 & 63,23 & 30,50 & 1,05 \\
\hline 12,13 & 30,50 & 1,44 & 61,22 & 6,10 & 1,38 \\
\hline 07,60 & 15,30 & 1,48 & 57,75 & 25,30 & 1,33 \\
\hline 01,40 & 40,75 & 1,24 & 57,30 & 2,3 & {$[1,98]$} \\
\hline 00,11 & 10,15 & 1,11 & 56,02 & 3,5 &, 59 \\
\hline $\mathbf{3 5 8 8 , 9 6}$ & 3,6 & 1,71 & 47,50 & 10,15 &, 66 \\
\hline 88,51 & 12,20 & 1,66 & 46,71 & 5,8 &, 88 \\
\hline 86,22 & 15,30 &, 75 & 43,69 & 10,15 &, 71 \\
\hline 77,74 & 5,8 & 1,16 & 40,70 & 2,3 & {$[1,35]$} \\
\hline 77,10 & 50,75 & 1,39 & 38,39 & 100,150 & 1,25 \\
\hline 75,89 & 15,25 & 1,35 & 30,73 & 40,40 & 1,32 \\
\hline 72,70 & 75,125 & 1,04 & 27,23 & $1+, 3$ & {$[1,76]$} \\
\hline 70,25 & 6,12 & 1,11 & 24,00 & 2,4 & 1,49 \\
\hline 66,30 & 15,25 & 1,23 & 19,76 & 3,6 &, 81 \\
\hline 65,61 & 10,20 & ,93 & 19,22 & 6,6 & 2,02 \\
\hline 59,23 & 6,10 &, 80 & 14,87 & 15,15 &, 86 \\
\hline 56,89 & 75,125 &, 94 & 10,44 & 30,25 & 1,24 \\
\hline 50,67 & 8,20 & 1,40 & 08,23 & 12,10 &, 52 \\
\hline 49,73 & 20,30 & 1,60 & 05,03 & 25,20 &, 98 \\
\hline 47,90 & 20,30 & 1,39 & 03,89 & 12,10 &, 60 \\
\hline 42,87 & 40,60 & 1,23 & 03,10 & 10,8 & 1,69 \\
\hline 39,17 & 5,8 & 1,77 & 3399,95 & $1+, 3$ & {$[1,61]$} \\
\hline 37,11 & 10,15 & 1,24 & 99,51 & 10,15 &, 63 \\
\hline 35,30 & 6,10 & 1,28 & 96,81 & 2,3 & 1,06 \\
\hline 33,35 & 12,20 & 1,83 & 94,96 & 3,5 & 1,76 \\
\hline 31,00 & 10,20 & 1,17 & 93,36 & 15,12 & 1,62 \\
\hline 27,58 & 15,25 & 1,19 & 92,20 & 75,75 & 1,26 \\
\hline
\end{tabular}




\begin{tabular}{|c|c|c|c|c|c|}
\hline$\lambda$ & $i$ & $\Delta \lambda / \lambda^{2}$ & $\lambda$ & $i$ & $\Delta \lambda / \lambda^{2}$ \\
\hline 3388,49 & 40,40 & $\mp 1,63$ & 3234,24 & 10,12 & $\mp 1,19$ \\
\hline 88,07 & 40,40 &, 75 & 31,89 & 40,50 & 1,71 \\
\hline 83,90 & 2,4 &, 89 & 29,00 & 15,20 & 1,37 \\
\hline 74,84 & 30,15 & 1,50 & 22,61 & 8,10 & 1,28 \\
\hline 73,61 & 15,8 & 1,04 & 14,35 & 40,50 & 1,70 \\
\hline 73,05 & 4,6 & 1,05 & 12,17 & 8,12 & 1,12 \\
\hline 70,73 & 4,6 & 1,52 & 3192,11 & 8,12 & 1,27 \\
\hline 61,35 & 4,6 & 1,15 & 91,31 & 4,6 &, 93 \\
\hline 60,61 & 4,6 & 1,50 & 83,08 & 30,40 & 1,14 \\
\hline 60,18 & 12,10 & 1,03 & 61,12 & 2,4 &, 94 \\
\hline 56,28 & 25,20 & 1,73 & 57,94 & 4,8 &, 96 \\
\hline 54,59 & 12,10 & 1,48 & 38,88 & 20,30 & 1,14 \\
\hline 49,59 & 4,4 & 1,12 & 37,08 & 2,4 & {$[1,43]$} \\
\hline 49,23 & 2,2 & 1,02 & 33,70 & 15,20 & 1,17 \\
\hline 45,00 & 15,15 & 1,14 & 32,22 & 2,4 & {$[1,09]$} \\
\hline 44,00 & $1+, 3$ & 1,18 & 29,96 & 20,30 & 1,01 \\
\hline 42,10 & $1+, 3$ & 1,09 & 29,38 & 20,30 &, 95 \\
\hline 27,05 & 15,12 & 1,26 & 26,10 & 15,20 &, 55 \\
\hline 19,19 & 8,6 & 1,63 & 25,33 & $1+, 3$ & {$[1,03]$} \\
\hline 11,53 & 3,3 & 1,73 & 20,90 & 4,8 & 1,32 \\
\hline $06 ; 48$ & 30,20 & 1,11 & 11,09 & 6,10 & 1,46 \\
\hline 05,33 & 25,25 & 1,39 & 06,79 & 25,40 & 1,32 \\
\hline 02,89 & 8,6 & 1,43 & 3055,00 & 15,25 & 1,20 \\
\hline 3288,99 & 12,10 & 1,92 & 36,57 & 8,12 & 1,55 \\
\hline 86,01 & 8,8 & 1,79 & 29,63 & 6,10 & 1,23 \\
\hline 85,89 & 2,2 & 1,78 & 28,18 & 10,15 & 1,00 \\
\hline 83,09 & 10,8 & 1,32 & 20,53 & 5,8 & 1,29 \\
\hline 80,92 & 2,2 &, 69 & 19,96 & $1+, 3$ & 1,19 \\
\hline 75,28 & 2,2 & 1,24 & 11,88 & 4,6 & 1,11 \\
\hline 74,14 & 4,3 & 1,46 & 03,88 & 6,10 & 1,37 \\
\hline 73,22 & 40,50 & 1,45 & 2985,53 & 4,6 &, 75 \\
\hline 69,81 & 4,4 & 1,38 & 81,18 & 3,5 & {$[1,60]$} \\
\hline 60,24 & 4,4 & 1,42 & 79,35 & 2,4 & 1,29 \\
\hline 50,62 & 10,4 & 1,98 & 78,21 & 4,6 & 1,55 \\
\hline 47,72 & 6,6 & 1,14 & 69,77 & 6,10 &, 56 \\
\hline 42,32 & 3,3 & 1,38 & 69,10 & 6,10 & 1,58 \\
\hline 41,26 & 20,20 & 1,14 & 62,81 & 5,10 & 1,05 \\
\hline 36,75 & 3,4 & 1,68 & 55,92 & 8,12 & 1,11 \\
\hline
\end{tabular}

Die Zirkontripletten sind auf Serien hin nicht genau untersucht worden. Solch eine Untersuchung von so vielen Linien würde eine große Menge Zeit in Anspruch nehmen. 
Die folgenden 29 Linien sind anscheinend nicht zerlegt.

\begin{tabular}{|c|c|c|c|c|c|}
\hline$\lambda$ & $i$ & $B$ & $\lambda$ & $i$ & $B$ \\
\hline 5508,5 & 8 & $D$ & 4030,26 & 8 & $\left.{ }^{3}\right)$ \\
\hline $\mathbf{5 4 8 5 , 4}$ & 8 & & 3989,27 & 2 & $n i$ \\
\hline 5136,0 & 10 & & 00,71 & 15 & 4) \\
\hline 4851,8 & 6 & $b D$ & 3781,80 & 8 & 5) $D$ \\
\hline 15,4 & 4 & $D$ & 24,94 & 5 & \\
\hline 4400,58 & 6 & $D^{1}$ ) & 06,79 & 5 & $D$ breit \\
\hline 4273,80 & 12 & $\left.{ }^{2}\right)$ & 3657,01 & 2 & 6) \\
\hline 66,96 & 4 & & 3549,90 & 5 & \\
\hline 50,89 & $1+$ & $D$ breit & 3431,71 & 8 & 2) \\
\hline 40,59 & 20 & $A$ & 3362,87 & 8 & 4) \\
\hline 13,45 & 5 & $A$ & 3354,08 & 3 & $\left.n i^{7}\right)$ \\
\hline 4181,13 & 8 & & 3314,70 & 15 & 2) \\
\hline 40,18 & 5 & $D$ etwas & 3131,23 & $1+$ & $D$ breit \\
\hline 28,08 & 12 & $A$ & 3013,44 & $1+$ & 8) \\
\hline 4044,80 & 20 & $A$ & & & \\
\hline
\end{tabular}

Die meisten dieser Linien sind, wie die Noten angeben, wahrscheinlich Linien eines anderen Typus, deren Komponenten zu kleine Zerlegung haben und zu diffus sind, um eine

1) Anzeichen eines schwachen Paares externer Komponenten.

2) $s$-Komponente starkes Zentrum, mit einem breiten Hintergrunde von Zerstreuung an beiden Seiten. $p$-Komponente breit.

3) $p$-Komponente verbreitert.

4) Breite Linie für s-Komponente, am schärfsten und stärksten an den Ecken mit weichem Zentrum, $p$-Komponente scharf.

5) Die wirklichen Komponenten können durch ein Carbonband verdeckt sein.

6) Möglicherweise getrennt, aber zu schwach.

7) Nicht identifiziert. Es kann kaum Exner und Hascheks Linie 3353,80 $\lambda$ sein.

8) $\mathrm{Zu}$ sehwach und diffus. Kann vielleisht Dublette sein. Zu weit im Violett für genauere Anaiyse. 
Analyse zu gestatten. Die mit $A$ bezeichneten Linien sind ziemlich sicher unzerlegt; und die, die nicht bezeichnet sind, sind wahrscheinlich unzerlegt. Die in der Bemerknng mit 2) bezeichneten Linien sind sehr eigentïmlich und wahrscheinlich gleichartig. Die Linien 5736,0, 4240,39, 3900,71 würden eine Serie bilden, aber das nächste Glied um 3750 herum und die folgenden Glieder sind nicht vorhanden.

VII. Osmium.

\begin{tabular}{r|r|r||r|r|r}
\hline \multicolumn{1}{c|}{$\lambda$} & $i$ & $\Delta \lambda / \lambda^{2}$ & \multicolumn{1}{c|}{$\lambda$} & $i$ & $\Delta \lambda / \lambda^{2}$ \\
\hline \hline 4420,63 & 25 & $\mp 1,20 s$ & 3794,05 & 4 & $\mp 1,29$ \\
& 25 &, $70 p$ & 90,28 & 6 & 1,83 \\
4395,08 & 4 & 1,49 & 82,37 & 12 & 1,82 \\
28,94 & 4 & 1,19 & 52,71 & 20 & 1,63 \\
11,15 & 6 &, 94 & 3598,26 & $2+$ & {$[1,55]$} \\
4294,17 & 8 & $\{1,18$ & 3561,04 & 8 & 1,35 \\
61,01 & 20 & 1,95 & 59,96 & 4 & 1,58 \\
12,02 & 12 & 1,72 & 28,76 & 5 & 1,61 \\
4175,74 & 5 & 1,49 & 3402,03 & 3 & 1,55 \\
73,42 & 10 & 1,44 & 3301,75 & 12 & 1,50 \\
35,95 & 15 & 1,97 & 3268,09 & 6 & 1,68 \\
12,17 & 10 & 1,65 & 3262,44 & 4 & 1,37 \\
4091,99 & 3 & 1,81 & 3156,38 & 4 & 1,62 \\
66,90 & 8 & 1,54 & 3058,77 & 8 & 1,55 \\
3977,38 & 6 & 1,54 & 2909,19 & 10 & 1,40 \\
63,79 & 8 & 1,59 & & & \\
3882,03 & 7 & 1,65 & & & \\
& & & & & \\
& & & & & \\
\end{tabular}

Osmium wurde als eine Substanz ausgewählt, die weniger Linien besitzt, wobei einige Typen sich durch einen großen Bereich des Spektrums wiederholen. Mit Ausnahme eines schwachen Quadruplett sind die Linien alle Triplette mit Zerlegungen zwischen ,70 und 1,97. Es gibt eine Anzahl von Paaren ähnlicher Zerlegung, aber nicht genug Vertreter für eine Serie. Wie man sieht, sind die Spektrallinien nicht stark; 
daher ist eine lange Exposition im magnetischen Felde erforderlich. Ein Charakteristikum der Komponenten ist ihre große Schärfe. Die nicht verzeichneten $p$-Komponenten haben sehr gleichförmig die doppelte Intensität der $s$-Komponenten.

\section{Vergleichung der Substanzen.}

Es ist wünschenswert, die oben behandelten Substanzen mit solchen früher beobachteten zu vergleichen, welche Serien zeigen und sich auch im Magnetfelde entsprechend verhalten. Die Komponenten der beiden Yttriumlinien 4358,91 und 3195,80 , die genaue Duplikate sind, wie man es bei Serien erwartet, entsprechen sechs von den neun Komponenten der Quecksilberlinie 5461 der zweiten Nebenserie. Das Quadruplett 4167,65 entspricht vier Komponenten des Bariumsextuplets 5854. Das Quadruplett 3982,75 entspricht vier Komponenten der dreizehn Komponenten der Quecksilberlinie 3663,5. Die übrigen Komponenten bei diesen drei Linien fehlen aber nicht deshalb, weil sie zu schwach sind, denn die betreffenden sind keine schwachen Linien.

Die zwei Zirkonlinien mit elf Komponenten haben zwei Paar Komponenten ähnlich zwei Paaren von Komponenten in der Neunkomponentenquecksilberlinie 5461. Sie haben zwei andere Paare von Komponenten, die in der Bariumbauptserie mit gröBerer Wellenlänge, Linie 4934, wiederkehren. Ihr eines übrigbleibendes Komponentenpaar findet sich in der Bariumhauptserie mit kleinerer Wellenlänge, Linie 4554. Das heiBt, die Komponenten dieser beiden Linien kommen in drei Serientypen vor. Es gibt noch andere Linien sowohl bei Yttrium wie bei Zirkon, für deren Komponenten derartige Parallelen aufgestellt werden können, wenn man mehr als eine Linie von Barium und Quecksilber benutzt. Solch ein Vergleich weist aber eher auf eine Differenz, als auf eine Identität im Charakter der Linien hin.

Die obigen Tabellen zeigen, daß die Abstände der Komponenten der Linien vielfache von Grundintervallen sind, sowie daB diese Intervalle sich häufig wiederholen. Verschiedene Linien sind nur dann vollständig identisch, wenn sie dasselbe 
Intervall, in demselben Vielfachen zeigen. Man darf sogar verlangen, daB die Komponenten auch dieselbe relative Intensität haben. Dies ist die Bedingung, die bei den bisher festgestellten Haupt- und Nebenserien erfüllt ist. Die Abstände in den Quadrupletten der Hauptserien sind das Vier- und Zweifache des Intervalles $0,37\left(\Delta \lambda / \lambda^{2}\right)$ für die Feldstärke 24400 und die der Sextuplette der Hauptserien sind das Fünf-, Drei- und Einfacbe desselben Wertes.

Während ich mit der Untersuchung dieser GesetzmäBigkeiten beschäftigt war, wurde eine Untersuchung, die sich mit der gleichen Frage befaßt, von C. Runge publiziert. ${ }^{1}$ ) Ich habe demgemäB das ganze Material, das $R$ unges Arbeiten und die vorstehenden Beobachtungen enthalten, im folgenden systematisch geordnet in einer Tabelle eingetragen, was die Verhältnisse klarer als jede wörtliche Beschreibung veranschaulicht. Ein konstantes Intervall, das mit den gleichen Zahlen multipliziert auftritt, bedingt einen Typus nach dem Prestonschen Gesetze. Von Runges Typen habe ich nur die aufgenommen, die bei meinen Messungen sich gleichfalls ergeben haben.

Der näheren Erklärung halber sei gesagt, daß der Wert ,$a^{6}$ in der folgenden Tabelle der Zerlegung von 1,11 für die vorausgesetzte Feldstärke entspricht; es ist dies die Zerlegung, die von C. Runge als die des ,normalen Tripletts" bezeichnet ist. Dieses Triplett gab ihm den Wert $1,75 \times 10^{7}$ für $e / m$ (Ladung durch $\mathrm{MaBe}$ ) in der Gleichung

$$
a=\Delta \lambda / \lambda^{2}=\frac{e}{m} \cdot \frac{H}{4 \pi c},
$$

$c$ ist die Geschwindigkeit des Lichtes und $I T$ die Feldstärke. Runge spricht dann folgendes allgemeine Gesetz aus:

„Die bisher beobachteten komplizierten Zerlegungen von Spektrallivien im magnetischen Felde zeigen die folgende Eigentümlichkeit: Die Abstände der Komponenten von der Mitte sind Vielfache eines aliquoten Teiles des normalen Ab-

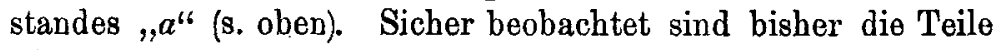
$a / 2, a / 3, a / 4, a / 5, a / 6, a / 7, a / 11, a / 12$.

1) C. Runge, Physik. Zeitschr. 8. p. 15. 1907. 
Tabelle der Intervalle.

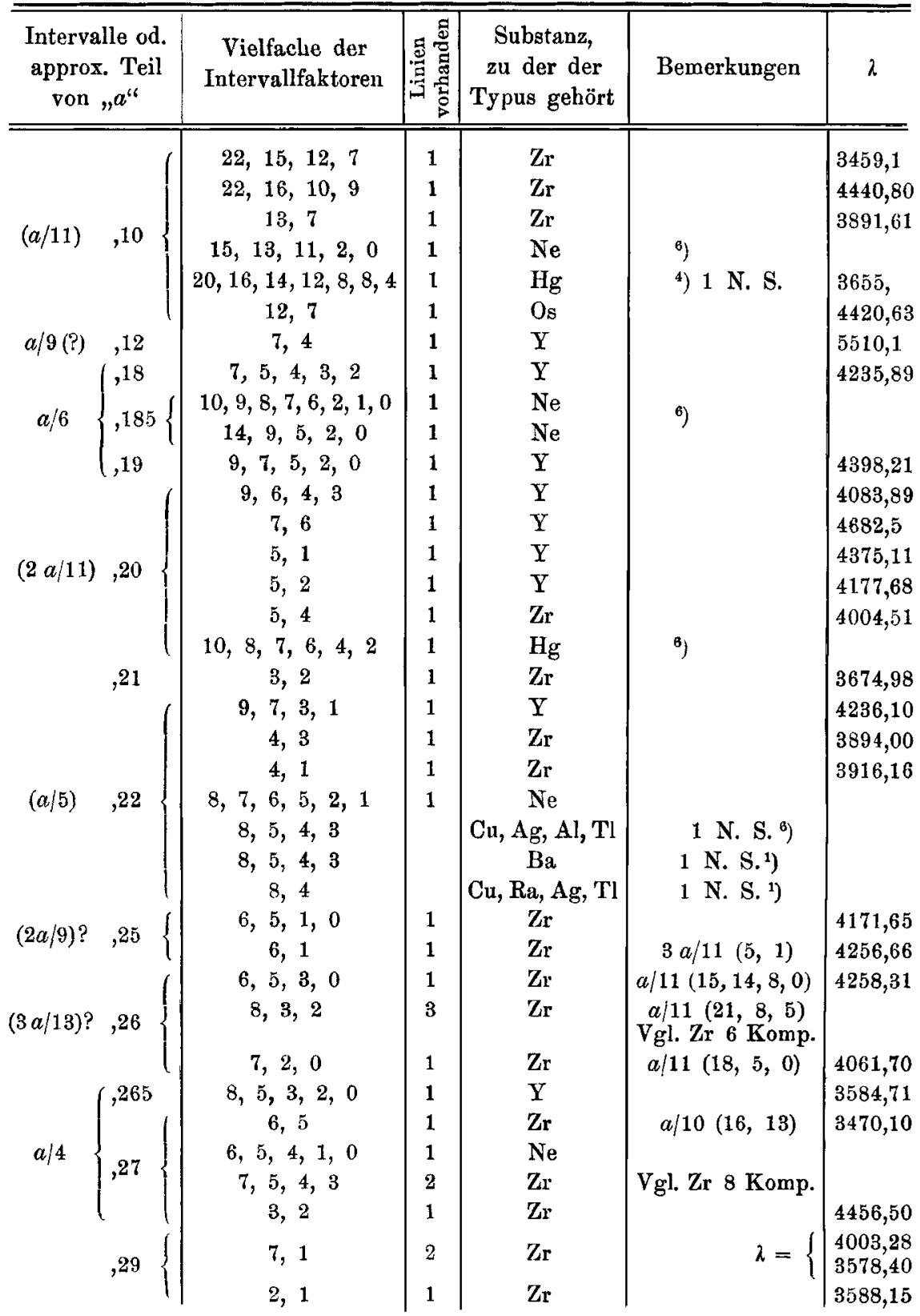




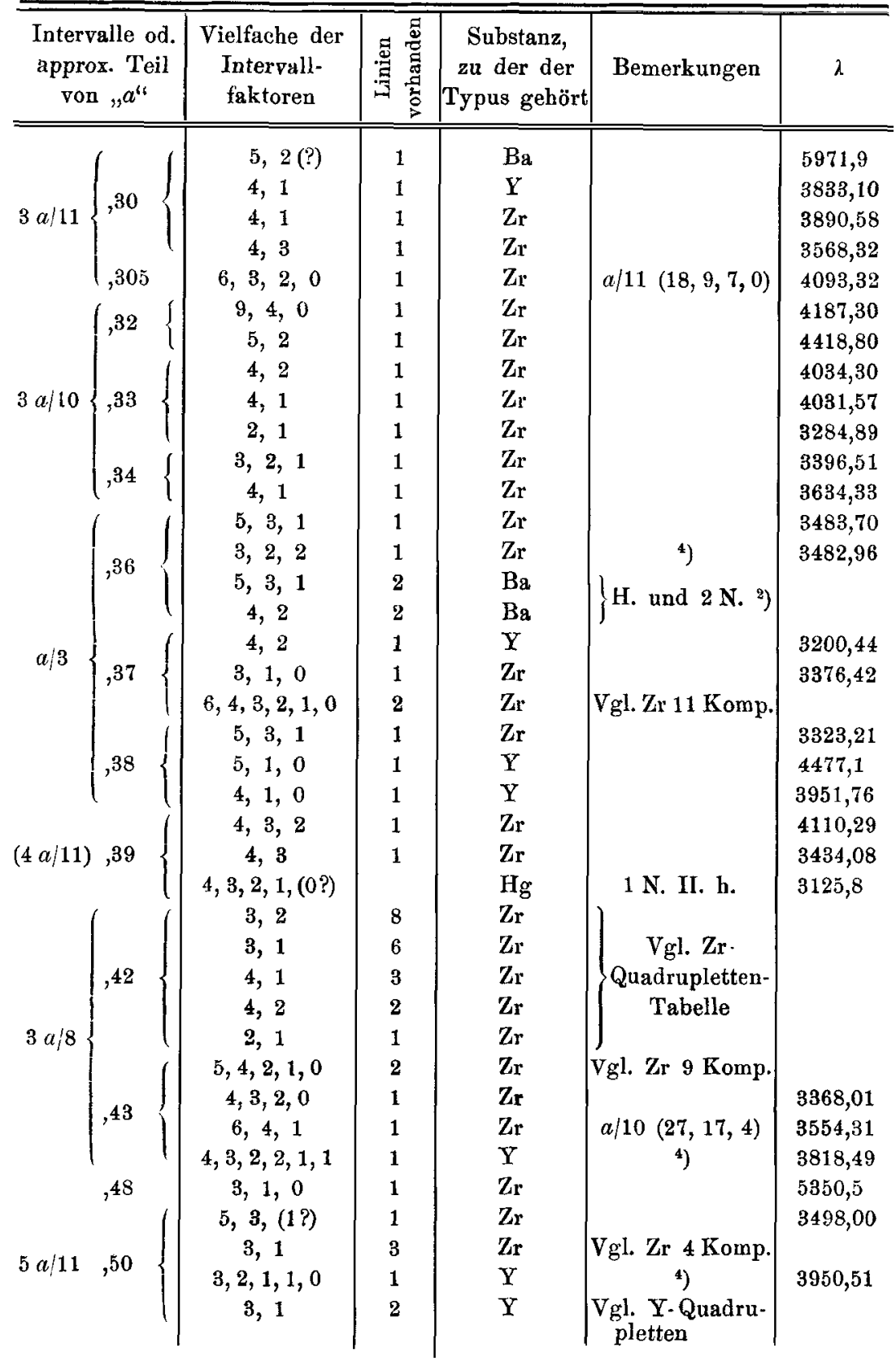




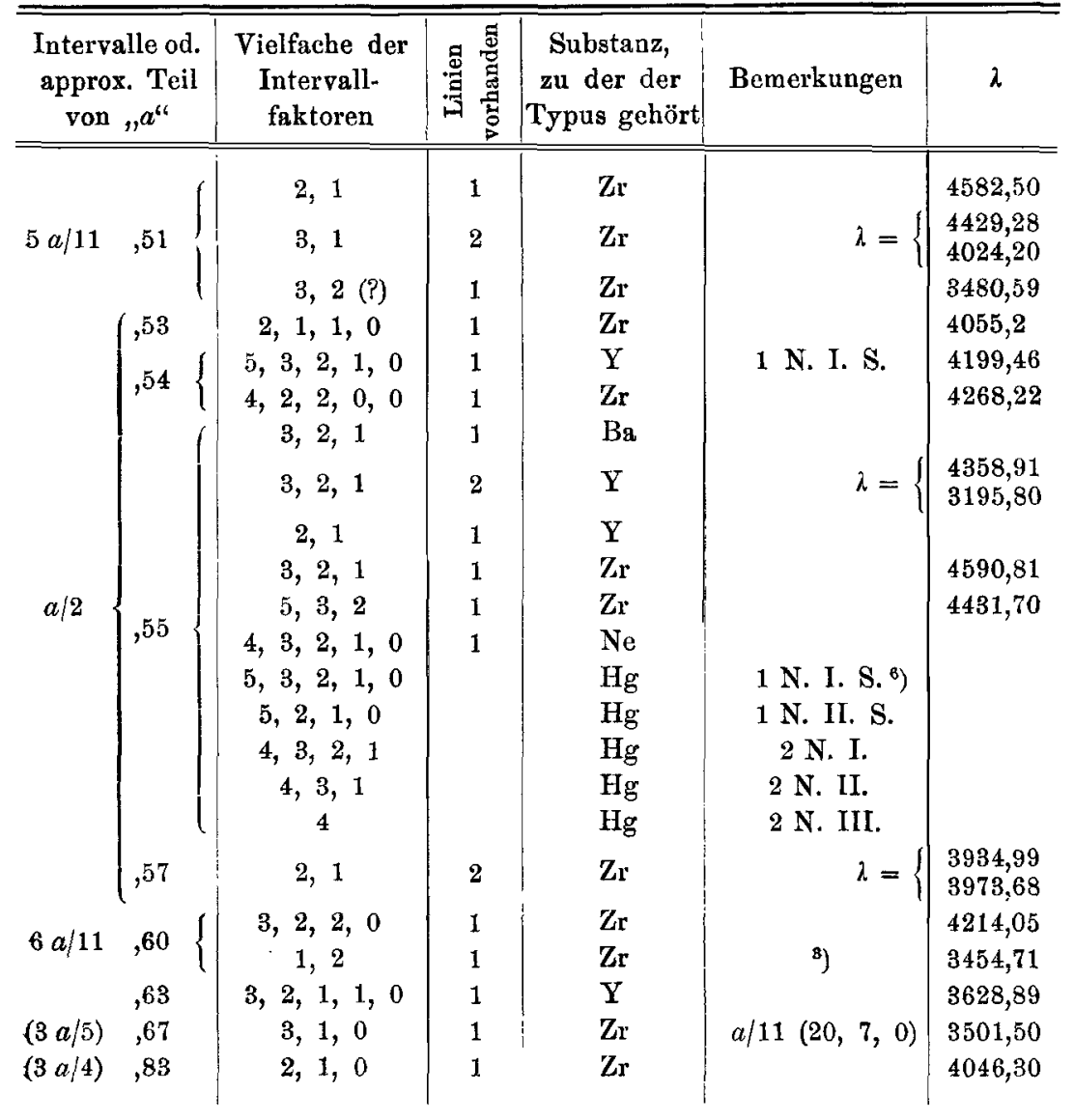

1) $\mathrm{Ba} 4166$ ist gerade so genau dargestellt durch $a / 4(6,4,3,2)$ und $\mathrm{Ba} 4436$, durch $a / 4(6,3)$. Keines von beiden System stellt sie genau dar. Vielfache von $(a / 11)$ stellen diese Linien sogar noch besser dar, ausgenommen für eine Komponente.

2) Serientypen, gezeigt durch $\mathrm{Na}, \mathrm{Cu}, \mathrm{Ag}, \mathrm{Al}, \mathrm{Pl}, \mathrm{Mg}, \mathrm{Ca}, \mathrm{Sr}, \mathrm{Ba}$.

3) Die $p$-Komponente liegt auBerhalb der $s$-Komponente.

4) Wenn $p$ - und $s$-Komponenten Duplikate in Stellung sind, sind sie bezeichnet durch wiederholende Zahlen.

5) Die Triplette dieser ersten Nebenserie sind 3 und $5 \mathrm{mal} a / 5$.

6) Untersucht von C. Runge, l. c. 
Bezüglich der Diskussion dieser Tabelle muB auf die Dissertation verwiesen werden.

\section{Schlußfolgeruungen.}

1. Die Linien der Tripletklasse bei Barium ordnen sich zumeist in Gruppen mit Abständen, die den Abständen $z$ wischen einigen Komponentensystemen von komplizierteren Typen nahe liegen.

2. Die groBe Mehrzahl der Abstände in Liniensystemen mit mehr als drei Komponenten stellen sich das als ganzzahlige Vielfache gewisser fundamentaler Intervalle, wobei die Faktoren kleine Zahlen sind. Die kleinsten Intervalle sind nach Runge aliquote Teile von „, $a^{\prime \prime}$, wobei „ $a^{\prime \prime}$ die Zerlegung der "normalen Triplette" ist. Runge fand kürzlich bei Neon die aliquoten Intervalle $a / 2, a / 3, a / 4, a / 5, a / 6, a, 7$, $a / 11, a / 12$. Alle, ausgenommen $a / 12$, sind auch bei der gegenwärtigen Untersuchung beobachtet worden.

3. Die große Mannigfaltigkeit in den magnetischen Zerlegungen ergibt sich hauptsächlich durch die Variation der Intervallfaktoren an Zahl und Größe; durch sie entstehen die verschiedenen Typen der Zerlegung.

4. Das Intervall $0,37(=a / 3)$ mal vier und zwei, sowie mal fünf, drei und eins, gibt die Quadruplett- bzw. Sextuplett. Haupt- und zweite Nebenserien, die von Runge und Paschen bei $\mathrm{Na}, \mathrm{Cu}, \mathrm{Ag}, \mathrm{Al}, \mathrm{Tl}, \mathrm{Mg}, \mathrm{Ca}, \mathrm{Sr}, \mathrm{Ba}$, Ra gefunden wurden. Das Quadruplett hat einen zweifelhaften Vertreter bei Yttrium, und das Sextuplett kommt bei einer Zirkonlinie vor. Indessen erscheint dasselbe Intervall kombiniert mit Faktoren in anderen Verhältnissen in neun neuen Typen. Einer der Typen von $\mathrm{Hg}$, erste Nebenserie, hat einen vereinzelten Vertreter bei diesen Substanzen; aber das gleiche Intervall tritt bei ihnen in mindestens 18 neuen Typen auf.

5. Die Spektren von Yttrium und Zirkon ergeben eine große Zahl neuer Typen.

6. Die hervorragendste Charakteristik der zahlreichen neuen Typen ist die Tatsache, daB sie sich innerhalb des mir zugänglichen Spektralbereiches nicht wiederholen. Es würde interessant sein, die Messungen weiter in das Ultraviolett hinein mit einem viel stärkeren Felde auszudehnen, um zu 
sehen, ob nicht doch Wiederholungen und sogar Serien vorkommen.

7. Das Intervall $0,42(=3 a / 8)$ bei Zirkon ist das einzige, das Serientypen zu versprechen scheint. Es liefert bei dieser Substanz acht Quadruplette von einem Typus und sechs von einem anderen; aber trotzdem ließen sich keine Serien finden.

8. Es gibt sechs Linien bei Yttrium, die mit sieben Linien bei Zirkon übereinstimmen und diese enthalten drei Typen. Dies sind kaum genug Übereinstimmungen, um eine Ähnlichkeit der Substanzen vermuten zu lassen, die indessen chemisch besteht. Die Substanzen sind parallele Glieder in zwei benachbarten (der dritten und vierten) Mendelejeff schen Gruppen.

9. Das eine Quadruplett bei Osmium hat ein für die erste Nebenserie charakteristisches Intervall; aber trotzdem ist es nicht von letzterem Typus.

10. Eine Untersuchung der Triplette auf Serien hin ist sehr umständlich. Es ist häufig von Vorteil, sie (wio beim Barium) in Gruppen zu teilen. Sie sehen dann wie kompliziertere Typen mit fehlenden Komponenten aus. Aber bei Yttrium und Zirkon würde man in Verlegenheit sein, zu wissen, welcher Typus zum Vergleich heranzuziehen wäre. Die Yttriumplatten sind sehr ausführlich auf Serien hin untersucht worden, ohne daB ein Ergebnis erzielt wäre. Bei Zirkon hat die Zeit das Studium von nur wenigen Triplettentypen gestattet. Die Resultate sind gleicherweise negativ ausgefallen. Dio in den Triplettenwerten auftretenden Abstände erstrecken sich über einen großen Raum. Sie gruppieren sich nicht um eine „normale Zerlegung" herum oder um Vielfache von aliquoten Teilen einer normalen Zerlegung.

11. Die unzerlegten Linien bei Zirkon zeigen keine Serien. Die meisten dieser Linien sind übrigens wahrscheinlich von komplizierterem Bau und nur mit den verfügbaren Mitteln nicht auflösbar.

12. Es gibt in den von mir untersuchten Spektren eine grobe Menge von Linien, die in Paaren angeordnet werden können. Solche Linien liegen meist verhältnismäBig nahe beieinander auf der Schwingungsskala. Die Paare können dieselbe Zerlegung haben oder nicht, und haben dieselbo oder 
eine verschiedene Zahl von Komponenten. Bei Substanzen, die Serien ergeben haben, sind solche Paare häufig gefunden. Diese Paare wiederholen sich dann in anderen Teilen des Spektrums mit beträchtlicher GleichmäBigkeit der Schwingungsdifferenzen. Bei Osmium sind keine Wiederholungen vorhanden, und bei Yttrium und Zirkon nur anscheinend, da die Abstände der Schwingungsdifferenz der anscheinenden Paare sebr unregelmäBig sind.

\section{Allgemeine Schlußfolgerung.}

Es besteht eine allgemeine Unähnlichkeit zwischen den Zerlegungen der Spektrallinien von Yttrium und Zirkon, sowie $z$ wischen diesen und den.Linien aller Substanzen, die Serien ergeben haben. Aber alle Substanzen haben gemeinsame Fundamentintervalle von kleiner GröBe und geringerer Zahl, die meist innig verbunden sind mit einer "normalen Zerlegung“"

Zum Schlusse möchte ich Hrn. Prof. Voigt, auf dessen Veranlassung hin diese Untersuchung unternommen wurde, für seine freundliche Beihilfe und begeisternde Ermutigung danken. In gleicher Weise bin ich Hrn. Prof. Runge zu Danke verpflichtet, der mir seine unschätzbare Erfabrung in Spektralarbeiten in liebenswürdiger Weise zur Verfügung gestellt hat.

(Eingegangen 19. Januar 1908.) · 